\title{
The Extended Invariance Principle for Signal Parameter Estimation in an Unknown Spatial Field
}

\author{
Felix Antreich, Member, IEEE, Josef A. Nossek, Fellow, IEEE, Gonzalo Seco-Granados, Senior Member, IEEE, \\ and A. Lee Swindlehurst, Fellow, IEEE
}

\begin{abstract}
This paper treats the problem of joint estimation of time-delay, Doppler frequency, and spatial (direction-of-arrival or DOA) parameters of several replicas of a known signal in an unknown spatially correlated noise field. Both spatially unstructured and structured data models have been proposed for this problem and corresponding maximum likelihood (ML) estimators have been derived. However, structured models require a high computational complexity and are sensitive to the antenna array response, while unstructured models are unable to achieve good performance in some scenarios. In this paper, it is shown how the extended invariance principle (EXIP) can be applied to obtain estimates with the quality of a spatially structured model, but with much lower complexity than directly utilizing a structured model and with greater robustness to errors in the model of the array response. EXIP improves the quality of the time-delay and Doppler frequency estimates obtained with a spatially unstructured model by introducing DOA estimates which are obtained in a second step through an innovative reparametrization. Simulation results for time-delay and Doppler frequency estimation for Global Positioning System (GPS) signals are presented and confirm that the proposed two-step approach attains the Cramer-Rao lower bound (CRLB) of the spatially structured model.
\end{abstract}

Index Terms-Antenna arrays, Cramer-Rao lower bound (CRLB), direction of arrival (DOA), Doppler frequency, extended invariance principle, high-resolution array signal processing, maximum likelihood estimation, multipath channel, propagation time-delay.

\section{INTRODUCTION}

C HANNEL estimation is important in many applications such as multiple input multiple output (MIMO) channel characterization, radar, synchronization, and for Global Navigation Satellite Systems (GNSS) including Global Positioning System (GPS). In this paper, we consider the problem of time-delay and Doppler frequency estimation of

Manuscript received August 06, 2010; revised November 25, 2010 and February 27, 2011; accepted March 22, 2011. Date of publication April 07, 2011; date of current version June 15,2011 . The associate editor coordinating the review of this manuscript and approving it for publication was Dr. Biao Chen.

F. Antreich is with the Institute for Communications and Navigation of the German Aerospace Center (DLR), Oberpfaffenhofen, 82235 Wessling, Germany (e-mail: felix.antreich@dlr.de).

J. A. Nossek is with the Institute for Circuit Theory and Signal Processing, Munich University of Technology (TUM), 80290 Munich, Germany (e-mail: josef.a.nossek@tum.de).

G. Seco-Granados is with the Department of Telecommunications and Systems Engineering, Universitat Autonoma de Barcelona (UAB), Bellaterra 08193 Barcelona, Spain (e-mail: gonzalo.seco@uab.cat).

A. L. Swindlehurst is with the Department of Electrical Engineering and Computer Science, University of California at Irvine (UCI), Irvine, CA 92697 USA (e-mail: swindle@uci.edu).

Digital Object Identifier 10.1109/TSP.2011.2140107 the line-of-sight signal (LOSS) of a GPS satellite in a multipath and radio frequency interference (RFI) environment. The quality of the ranging data provided by a GNSS receiver largely depends on the synchronization error, that is, on the accuracy of the estimation of the propagation time-delay of the LOSS. When the LOSS is corrupted by several superimposed delayed replicas (reflective multipath) and/or additional RFI, the estimation of the propagation time-delay (and thus the positioning) can be severely degraded in state-of-the-art GNSS receivers [1]-[3]. In this paper, we consider the general case where the spatial components of the RFI are unknown. Several techniques have been proposed in the literature for solving the multipath problem in GNSS using one antenna, see, e.g., [4]-[7]. For joint multipath and RFI mitigation, interesting approaches using antenna arrays have been developed in recent years [8]-[10].

Spatially unstructured data models have been used for such problems in order to achieve low complexity and robustness to array response modeling errors [11] and a solution for RFI with an unknown spatial field was given in [12]. On the other hand a spatially structured data model provides better results [13]-[15], but entails rather high complexity in the parameter estimation.

In a spatially unstructured model, the impinging wavefront is simply described by a vector spatial signature without further parameterization. On the other hand, a spatially structured data model parameterizes the wavefront in terms of its complex amplitude and direction of arrival (DOA) based on a model for the response of the array. This obviously requires detailed knowledge of the antenna response and the array geometry, while using a spatially unstructured data model provides the advantage of robustness to errors in array response model, its geometry and other hardware biases [16].

Even if the antenna array response can be approximately determined, the true antenna array response can be significantly different due for example to changes in antenna location, temperature, calibration inaccuracy and the surrounding environment. The effects of such model errors have been studied in [17]-[20] among many others. In order to account for these effects and thus exploit the full resolution capabilities of a spatially structured data model, a generalized model for the response of the antenna array needs to be considered [21]-[23], which introduces even more complexity in the parameter estimation. These methods are referred to as autocalibration techniques.

In [16] it was shown how the extended invariance principle (EXIP) can be applied to refine maximum likelihood (ML) estimates of a spatially unstructured data model to achieve the per- 
formance available using a spatially structured data model. The EXIP, which was first introduced in [24], involves reparameterizing the ML criterion in such a way that it admits an approximate initial solution (much simpler) which is then refined by means of a weighted least squares (WLS) fit. The optimal weighting is the Fisher information matrix (FIM) corresponding to the unstructured ML criterion. Furthermore, it is also shown in [16] that the overall estimation performance of the proposed approach can be improved by taking into account array calibration errors in the estimation of the signal parameters. In particular, it is demonstrated that information about the second-order statistics of the errors of the array response model can be used to "regularize" the EXIP solution and make it more robust to such errors.

This paper extends and generalizes the approach given in [16] to a multipath case in an unknown spatial field (e.g., noise plus RFI). We present a two-step approach that improves the quality of ML estimates which are obtained with a spatially unstructured model in order to derive ML estimates for a spatially structured model. The two steps are:

1) Apply the space alternating generalized expectation maximization (SAGE) algorithm to obtain ML estimates for an unstructured model.

2) Adopt the EXIP to refine the ML estimates obtained for an unstructured model in order to derive time-delay and Doppler frequency ML estimates for a structured data model.

The WLS fit in the second step involves a search only for the DOAs of the impinging wavefronts. We will prove that the resulting DOA estimation problem is equivalent to a complex augmented subspace fitting (SSF) problem. As a solution for the special case of a uniform linear array (ULA), we derive an innovative reparametrization approach based on the iterative quadratic maximum likelihood (IQML) algorithm [25] that exploits the special structure of the defined complex augmented SSF problem. This novel low-complexity approach reduces to iteratively solving a quadratic problem with linear constraints. The DOA estimates can be derived explicitly in closed-form since the reparametrization of the DOA estimation problem leads to finding the simple root of several first-order monic polynomials.

In the proposed two-step approach only the second step involves a search for the DOAs, thus it is only in this second step that errors in the array response model need to be considered. In order to achieve robustness of the EXIP to errors in the array response model, a novel "regularization" of the weighting matrix of the WLS is defined based on a "regularized" Schur complement. This regularization approach avoids the application of autocalibration techniques as discussed above, which would introduce additional complexity to the parameter estimation process.

There are only few publications that treat the general and complex problem considered in this work involving all of the parameters, e.g., [13]-[15], [26]. Most of these publications only consider white noise, but not unknown spatially colored interference or errors in the array antenna model as done in this work. Hence, the important contribution of this paper is to propose a computationally tractable solution that additionally is able to account for an unknown spatial field and imprecision in the array calibration. The proposed two-step EXIP approach is the key to achieving these goals.

The paper is organized as follows. In Section II, the unstructured and structured data models are introduced. Section III describes the first step of our proposed two-step approach, in which the SAGE algorithm is used for ML parameter estimation for the unstructured model. In Section IV, the second step of our proposed two-step approach is described. The EXIP is briefly introduced, it is proven that the resulting DOA estimation problem is equivalent to a complex augmented SSF problem and an innovative reparametrization is developed for DOA estimation. In Section $\mathrm{V}$ we discuss the reduction of computational complexity achieved by our approach. In Section VI, the behavior of the proposed two-step approach is described using several simulation examples. Finally, in Section VII, we will present our conclusions.

\section{DATA MODEL}

We assume that $L$ narrowband planar wavefronts, $1 \leq \ell \leq L$ are impinging on an array of $M$ sensor elements. The noise-plus-interference corrupted baseband signal at the antenna output $\mathbf{y}(t) \in \mathbb{C}^{M \times 1}$ can be modelled as a superposition of $L$ wavefronts $\mathbf{s}_{\ell}(t) \in \mathbb{C}^{M \times 1}$ and temporally white complex Gaussian noise $\mathbf{n}(t) \in \mathbb{C}^{M \times 1}$, with zero-mean and unknown spatial covariance matrix $\mathbf{Q} \in \mathbb{C}^{M \times M}$. In the following we will define spatially unstructured and structured models. We assume that the number of impinging wavefronts $L$ is the same for the spatially unstructured and structured models.

\section{A. Structured Model}

The complex baseband signal received by the antenna array is

$$
\mathbf{y}(t)=\mathbf{s}(t)+\mathbf{n}(t)=\sum_{\ell=1}^{L} \mathbf{s}_{\ell}(t)+\mathbf{n}(t)
$$

where $\mathbf{s}(t) \in \mathbb{C}^{M \times 1}$ denotes the superimposed signal replicas

$$
\mathbf{s}_{\ell}(t)=\mathbf{a}\left(\phi_{\ell}\right) \gamma_{\ell} \mathrm{e}^{\mathrm{j} 2 \pi \nu_{\ell} t} c\left(t-\tau_{\ell}\right)
$$

$\mathbf{a}\left(\phi_{\ell}\right) \in \mathbb{C}^{M \times 1}$ defines the steering vector of the antenna array and $c\left(t-\tau_{\ell}\right)$ denotes a known signal waveform $c(t)$ with unknown delay $\tau_{\ell}$. We define the parameter vector

$$
\boldsymbol{\theta}=\left[\operatorname{Re}\{\boldsymbol{\gamma}\}^{\mathrm{T}}, \operatorname{Im}\{\boldsymbol{\gamma}\}^{\mathrm{T}}, \boldsymbol{\phi}^{\mathrm{T}}, \boldsymbol{\tau}^{\mathrm{T}}, \boldsymbol{\nu}^{\mathrm{T}}\right]^{\mathrm{T}} \in \mathcal{D}_{\boldsymbol{\theta}}
$$

composed of the vector of complex amplitudes $\gamma=$ $\left[\gamma_{1}, \ldots, \gamma_{\ell}, \ldots, \gamma_{L}\right]^{\mathrm{T}}$, the vector of azimuth angles $\boldsymbol{\phi}=$ $\left[\phi_{1}, \ldots, \phi_{\ell}, \ldots, \phi_{L}\right]^{\mathrm{T}}$, the vector of time-delays $\boldsymbol{\tau}=$ $\left[\tau_{1}, \ldots, \tau_{\ell}, \ldots, \tau_{L}\right]^{\mathrm{T}}$ and the vector of Doppler frequencies $\boldsymbol{\nu}=\left[\nu_{1}, \ldots, \nu_{\ell}, \ldots, \nu_{L}\right]^{\mathrm{T}}$ corresponding to each wavefront. In (3) the set $\mathcal{D}_{\boldsymbol{\theta}}$ denotes the domain of the parameter vector $\boldsymbol{\theta}$. The parameters of one wavefront are collected in the parameter vector

$$
\boldsymbol{\theta}_{\ell}=\left[\operatorname{Re}\left\{\gamma_{\ell}\right\}, \operatorname{Im}\left\{\gamma_{\ell}\right\}, \phi_{\ell}, \tau_{\ell}, \nu_{\ell}\right]^{\mathrm{T}} .
$$

The spatial observations are collected at $N$ time instants, as $\mathbf{y}[n]=\mathbf{y}\left(n \cdot T_{s}\right)$ with $n=1,2, \ldots, N$, where $T_{s}$ is the sample 
interval and $f_{s}=\frac{1}{T_{s}}$ denotes the sampling frequency. The channel parameters are assumed constant during the observation interval. Collecting the samples of the observation interval leads to

$$
\begin{aligned}
\mathbf{Y} & =[\mathbf{y}[1], \mathbf{y}[2], \ldots, \mathbf{y}[N]] \in \mathbb{C}^{M \times N} \\
\mathbf{N} & =[\mathbf{n}[1], \mathbf{n}[2], \ldots, \mathbf{n}[N]] \in \mathbb{C}^{M \times N} \\
\mathbf{S}(\boldsymbol{\theta}) & =[\mathbf{s}[1], \mathbf{s}[2], \ldots, \mathbf{s}[N]] \in \mathbb{C}^{M \times N} \\
\mathbf{S}_{\ell}\left(\boldsymbol{\theta}_{\ell}\right) & =\left[\mathbf{s}_{\ell}[1], \mathbf{s}_{\ell}[2], \ldots, \mathbf{s}_{\ell}[N]\right] \in \mathbb{C}^{M \times N} .
\end{aligned}
$$

Thus, the signal for the structured model can be written in matrix notation as

$$
\begin{aligned}
\mathbf{Y} & =\mathbf{S}(\boldsymbol{\theta})+\mathbf{N}=\sum_{\ell=1}^{L} \mathbf{S}_{\ell}\left(\boldsymbol{\theta}_{\ell}\right)+\mathbf{N} \\
& =\mathbf{A} \boldsymbol{\Gamma}(\mathbf{C} \odot \mathbf{D})+\mathbf{N}
\end{aligned}
$$

where $\odot$ denotes the Hadamard-Schur product,

$$
\begin{aligned}
\mathbf{A} & =\left[\mathbf{a}\left(\phi_{1}\right) \cdots \mathbf{a}\left(\phi_{\ell}\right) \cdots \mathbf{a}\left(\phi_{L}\right)\right] \in \mathbb{C}^{M \times L} \\
\mathbf{\Gamma} & =\operatorname{diag}\{\boldsymbol{\gamma}\} \in \mathbb{C}^{L \times L} \\
\mathbf{C} & =\left[\mathbf{c}\left(\tau_{1}\right) \cdots \mathbf{c}\left(\tau_{\ell}\right) \cdots \mathbf{c}\left(\tau_{L}\right)\right]^{\mathrm{T}} \in \mathbb{R}^{L \times N}
\end{aligned}
$$

contains the sampled and shifted waveform $c(t)$ for each impinging wavefront

$$
\mathbf{c}\left(\tau_{\ell}\right)=\left[c\left(T_{s}-\tau_{\ell}\right), \ldots, c\left(n T_{s}-\tau_{\ell}\right), \ldots, c\left(N T_{s}-\tau_{\ell}\right)\right]^{\mathrm{T}}
$$

and

$$
\mathbf{D}=\left[\mathbf{d}\left(\nu_{1}\right) \cdots \mathbf{d}\left(\nu_{\ell}\right) \cdots \mathbf{d}\left(\nu_{L}\right)\right]^{\mathrm{T}} \in \mathbb{C}^{L \times N}
$$

contains the complex exponential functions conveying the Doppler frequency of each wavefront

$$
\mathrm{d}\left(\nu_{\ell}\right)=\left[\mathrm{e}^{\mathrm{j} 2 \pi \nu_{\ell} T_{s}}, \ldots, \mathrm{e}^{\mathrm{j} 2 \pi \nu_{\ell} n T_{s}}, \ldots, \mathrm{e}^{\mathrm{j} 2 \pi \nu_{\ell} N T_{s}}\right]^{\mathrm{T}} .
$$

\section{B. Unstructured Model}

Alternatively, for the simpler unstructured model we write

$$
\mathbf{s}_{\ell}(t)=\mathbf{h}_{\ell} \mathrm{e}^{\mathrm{j} 2 \pi \nu_{\ell} t} c\left(t-\tau_{\ell}\right)
$$

where explicit parametrization on the DOA and signal amplitude is eliminated in favor of an unstructured spatial signature $\mathbf{h}_{\ell} \in \mathbb{C}^{M \times 1}$. We define the parameter vector

$$
\boldsymbol{\xi}=\left[\operatorname{Re}\{\operatorname{vec}\{\mathbf{H}\}\}^{\mathrm{T}}, \operatorname{Im}\{\operatorname{vec}\{\mathbf{H}\}\}^{\mathrm{T}}, \boldsymbol{\tau}^{\mathrm{T}}, \boldsymbol{\nu}^{\mathrm{T}}\right]^{\mathrm{T}} \in \mathcal{D}_{\boldsymbol{\xi}}
$$

with

$$
\mathbf{H}=\left[\mathbf{h}_{1} \cdots \mathbf{h}_{\ell} \cdots \mathbf{h}_{L}\right] \in \mathbb{C}^{M \times L} .
$$

In (17), vec $\{\cdot\}$ denotes the vec operator, which vectorizes a matrix by stacking its columns. The set $\mathcal{D}_{\xi}$ denotes the domain of the parameter vector $\boldsymbol{\xi}$. The parameters of each wavefront are collected in the parameter vector

$$
\boldsymbol{\xi}_{\ell}=\left[\operatorname{Re}\left\{\mathbf{h}_{\ell}\right\}^{\mathrm{T}}, \operatorname{Im}\left\{\mathbf{h}_{\ell}\right\}^{\mathrm{T}}, \tau_{\ell}, \nu_{\ell}\right]^{\mathrm{T}} .
$$

The signal for the unstructured model can be written in matrix notation by introducing $\boldsymbol{\xi}$ and $\boldsymbol{\xi}_{\ell}$ to (9). In general $\mathbf{H}=\mathbf{A} \Gamma$, but the unstructured model makes no assumptions about the elements or the structure of $\mathbf{H}$.

\section{PARAmeter ESTIMATION FOR THE UnStRUCTURED MODEL}

In this section we discuss the first step of the proposed twostep approach in which the parameters of the unstructured model are estimated.

\section{A. Maximum Likelihood (ML) Estimation}

Assuming temporally white complex Gaussian noise $\mathbf{n}(t)$ with zero-mean and unknown spatial covariance matrix $\mathbf{Q} \in \mathbb{C}^{M \times M}$, the log-likelihood function for the unstructured data model can be written as

$$
\begin{aligned}
l(\mathbf{Y} ; \boldsymbol{\eta})= & l\left(\mathbf{Y} ; \boldsymbol{\xi}, \operatorname{Re}\left\{\mathbf{J}_{M}^{R} \operatorname{vec}\{\mathbf{Q}\}\right\}^{\mathrm{T}}, \operatorname{Im}\left\{\mathbf{J}_{M}^{I} \operatorname{vec}\{\mathbf{Q}\}\right\}^{\mathrm{T}}\right) \\
= & -M N \ln (\pi)-N \ln (\operatorname{det}(\mathbf{Q})) \\
& -\operatorname{tr}\left(\mathbf{Q}^{-1}(\mathbf{Y}-\mathbf{S}(\boldsymbol{\xi}))(\mathbf{Y}-\mathbf{S}(\boldsymbol{\xi}))^{\mathrm{H}}\right)
\end{aligned}
$$

where

$$
\boldsymbol{\eta}=\left[\boldsymbol{\xi}^{\mathrm{T}}, \operatorname{Re}\left\{\mathbf{J}_{M}^{R} \operatorname{vec}\{\mathbf{Q}\}\right\}^{\mathrm{T}}, \operatorname{Im}\left\{\mathbf{J}_{M}^{I} \operatorname{vec}\{\mathbf{Q}\}\right\}^{\mathrm{T}}\right]^{\mathrm{T}}
$$

$(\cdot)^{\mathrm{H}}$ denotes complex conjugate transposition, $\operatorname{det}(\cdot)$ is the determinant operation, $\operatorname{tr}(\cdot)$ is the trace operator, $\mathbf{J}_{M}^{R}$ is a selection matrix with dimensions $\frac{M^{2}+M}{2} \times M^{2}$ which selects the upper-triangular elements of $\mathbf{Q}$ from $\operatorname{vec}\{\mathbf{Q}\}$, and $\mathbf{J}_{M}^{I}$ is a selection matrix with dimensions $\frac{M^{2}-M}{2} \times M^{2}$ that selects the lower-triangular elements of $\mathbf{Q}$ excluding the diagonal from $\operatorname{vec}\{\mathbf{Q}\}$.

The maximum likelihood estimator (MLE) is given by

$$
\hat{\boldsymbol{\eta}}=\arg \max _{\boldsymbol{\eta}}\{l(\mathbf{Y} ; \boldsymbol{\eta})\} .
$$

The ML estimate of $\mathbf{Q}$ can be derived as [16]

$$
\hat{\mathbf{Q}}=\frac{1}{N}(\mathbf{Y}-\mathbf{S}(\boldsymbol{\xi}))(\mathbf{Y}-\mathbf{S}(\boldsymbol{\xi}))^{\mathrm{H}} .
$$

\section{B. Space-Alternating Generalized Expectation Maximization (SAGE) Algorithm}

In order to obtain the ML estimates $\hat{\xi}$ for the unstructured model (22) we apply the iterative SAGE algorithm [27], [28]. Instead of directly performing a high-dimensional nonlinear optimization procedure, the SAGE algorithm provides a sequential approximation of the MLE by performing a sequence of maximization steps in spaces of lower dimension, thereby considerably reducing the complexity. In general, there are only a small number of algorithms available, which are used to solve such high-dimensional nonlinear problems in channel estimation, e.g., [13], [14], and [26]. The SAGE algorithm is very suited for such parameter estimation problems, in particular when both time-delays and Doppler frequencies of several superimposed signals have to be estimated [13], [15], [27]. 
We assume that the number of wavefronts $L$ is known. In order to determine $L$, several methods can be applied, e.g., simple rank tests on $\mathbf{Y}$, methods using Akaike information criterion (AIC) [29] or methods using the minimum description length (MDL) criterion [30], [31], or other methods given in [16] and [32]. In case of unknown colored noise fields, methods as described in [33] and [34] can be applied. Tracking the parameter estimates and the model order $L$ can be performed by methods as the SAGE Kalman filter approach developed in [35].

The basic concept of the SAGE algorithm is the hidden data space [27]. In our case, we choose the hidden data space as $\mathbf{X}_{\ell}=\mathbf{S}_{\ell}+\mathbf{N}_{\ell}$, where $\mathbf{N}_{\ell}$ is temporally white complex Gaussian noise with covariance $\beta_{\ell} \mathbf{Q}$, where $\sum_{\ell=1}^{L} \beta_{\ell}=1$ and $\mathbf{N}=$ $\sum_{\ell=1}^{L} \mathbf{N}_{\ell}$. The stochastic mapping of the hidden data space to the observable signal is $\mathbf{Y}=\mathbf{X}_{\ell}+\sum_{\substack{\ell^{\prime}=1 \\ \ell^{\prime} \neq \ell}}^{L} \mathbf{S}_{\ell^{\prime}}+\mathbf{N}_{\ell^{\prime}}$. The SAGE algorithm performs two steps, the expectation step (E-step) and the maximization step (M-step). Within the E-step an estimate of the unobservable data, namely the hidden data space $\hat{\mathbf{X}}_{\ell}$ and the spatial covariance matrix $\hat{\mathbf{Q}}$, is derived based on the current update of the estimates of the parameters $\hat{\boldsymbol{\xi}}$ and the observable data $\mathbf{Y}$. In the M-step the estimates of the signal parameters are updated.

Thus, the E-step can be formulated as

$$
\begin{aligned}
\hat{\mathbf{X}}_{\ell} & =\mathbf{Y}-\sum_{\substack{\ell^{\prime}=1 \\
\ell^{\prime} \neq \ell}}^{L} \mathbf{S}_{\ell^{\prime}}\left(\hat{\boldsymbol{\xi}}_{\ell^{\prime}}\right) \\
\hat{\mathbf{Q}} & =\frac{1}{N}(\mathbf{Y}-\mathbf{S}(\hat{\boldsymbol{\xi}}))(\mathbf{Y}-\mathbf{S}(\hat{\boldsymbol{\xi}}))^{\mathrm{H}} .
\end{aligned}
$$

The M-Step then can be given as

$$
\begin{aligned}
& \hat{\tau}_{\ell}=\arg \max _{\tau_{\ell}}\left\{\left\|\hat{\mathbf{Q}}^{-\frac{1}{2}} \hat{\mathbf{X}}_{\ell}\left(\mathbf{c}\left(\tau_{\ell}\right) \odot \mathbf{d}\left(\hat{\nu}_{\ell}\right)\right)^{*}\right\|_{2}^{2}\right\} \\
& \hat{\nu}_{\ell}=\arg \max _{\nu_{\ell}}\left\{\left\|\hat{\mathbf{Q}}^{-\frac{1}{2}} \hat{\mathbf{X}}_{\ell}\left(\mathbf{c}\left(\hat{\tau}_{\ell}\right) \odot \mathbf{d}\left(\nu_{\ell}\right)\right)^{*}\right\|_{2}^{2}\right\} \\
& \hat{\mathbf{h}}_{\ell}=\hat{\mathbf{X}}_{\ell}\left(\mathbf{c}\left(\hat{\tau}_{\ell}\right) \odot \mathbf{d}\left(\hat{\nu}_{\ell}\right)\right)^{+} .
\end{aligned}
$$

$(\cdot)^{+}$in $(28)$ denotes the Moore-Penrose pseudoinverse. The E-step and the M-step (24)-(28) are performed iteratively wavefront by wavefront with $\ell=1, \ldots, L$ until the algorithm converges.

Initialization of the SAGE algorithm is performed by successive interference cancellation starting with $\hat{\boldsymbol{\xi}}=[0, \ldots, 0]^{\mathrm{T}}$ as described in [15]. For the covariance matrix $\mathbf{Q}$ the initial estimate is

$$
\hat{\mathbf{Q}}=\frac{1}{N} \mathbf{Y} \mathbf{Y}^{\mathrm{H}}
$$

\section{EXTENDED INVARIANCE PRINCIPLE (EXIP)}

In this section, we describe the second step of our approach, in which the initial unstructured estimates are updated for the structured model.

We apply EXIP in order to estimate $\boldsymbol{\theta}$ from an estimate of $\boldsymbol{\xi}$. The estimation of the deterministic parameters of the wavefronts ( $\boldsymbol{\xi}$ or $\boldsymbol{\theta}$ ) is decoupled from estimating the noise parameters $(\mathbf{Q})$, as the related FIM is block diagonal. Further, the parametrization of $\mathbf{Q}$ is the same for both the spatially structured and unstructured models. Thus, the EXIP approach can be described as follows [16], [24].

Theorem 1: Assuming that there exists a mapping function $f$ which is one-to-one and well defined in $\mathcal{D}_{\boldsymbol{\theta}}$ and which satisfies

$$
\boldsymbol{\xi}=\boldsymbol{f}(\boldsymbol{\theta}) \in \mathcal{D}_{\boldsymbol{\xi}}, \quad \forall \boldsymbol{\theta} \in \mathcal{D}_{\boldsymbol{\theta}}
$$

and

$$
\lim _{N \rightarrow \infty} \hat{\boldsymbol{\xi}}=\lim _{N \rightarrow \infty} \boldsymbol{f}(\hat{\boldsymbol{\theta}})
$$

then

$$
\hat{\boldsymbol{\theta}}=\arg \min _{\boldsymbol{\theta}}[\hat{\boldsymbol{\xi}}-\boldsymbol{f}(\boldsymbol{\theta})]^{\mathrm{T}} \mathbf{W}[\hat{\xi}-\boldsymbol{f}(\boldsymbol{\theta})]
$$

is asymptotically (for large $N$ ) equivalent to the structured $\mathrm{ML}$ estimate $\hat{\boldsymbol{\theta}}$, where

$$
\mathbf{W}=\left.\mathrm{E}\left[\frac{\partial^{2} l(\mathbf{Y} ; \boldsymbol{\eta})}{\partial \boldsymbol{\xi} \partial \boldsymbol{\xi}^{\mathrm{T}}}\right]\right|_{\boldsymbol{\xi}=\hat{\boldsymbol{\xi}}}
$$

Proof: A proof can be found in [16], [24].

The weighting matrix $\mathbf{W} \in \mathbb{R}^{(2 L M+2 L) \times(2 L M+2 L)}$ can be found from the block of the FIM that is related to the signal parameters $\boldsymbol{\xi}$. The weighting matrix $\mathbf{W}$ is derived in Appendix A.

The EXIP as given in (32) determines $\hat{\hat{\theta}}$ such that the point $\boldsymbol{f}(\boldsymbol{\theta}) \in \mathcal{D}_{\boldsymbol{\xi}}$ is as close to $\hat{\boldsymbol{\xi}}$ as possible in the metric induced by $\mathbf{W}$ [24]. The mapping function $\boldsymbol{f}(\boldsymbol{\theta})$ is given as

$$
\boldsymbol{f}(\boldsymbol{\theta})=\left[\begin{array}{c}
\boldsymbol{\Psi}(\boldsymbol{\phi})\left[\begin{array}{l}
\operatorname{Re}\{\boldsymbol{\gamma}\} \\
\operatorname{Im}\{\boldsymbol{\gamma}\}
\end{array}\right] \\
\boldsymbol{\nu}
\end{array}\right]
$$

where

$$
\Psi(\phi)=\left[\begin{array}{cc}
\operatorname{Re}\left\{\mathbf{I}_{L} \square \mathbf{A}\right\} & -\operatorname{Im}\left\{\mathbf{I}_{L} \square \mathbf{A}\right\} \\
\operatorname{Im}\left\{\mathbf{I}_{L} \square \mathbf{A}\right\} & \operatorname{Re}\left\{\mathbf{I}_{L} \square \mathbf{A}\right\}
\end{array}\right]
$$

and $\square$ denotes the Khatri-Rao product [36, p. 1355]. Minimizing (32) with respect to $\boldsymbol{\tau}$ and $\boldsymbol{\nu}$ leads to the refined estimates

$$
\begin{aligned}
{\left[\begin{array}{c}
\hat{\boldsymbol{\tau}} \\
\hat{\hat{\boldsymbol{\nu}}}
\end{array}\right]=\left[\begin{array}{c}
\hat{\boldsymbol{\tau}} \\
\hat{\boldsymbol{\nu}}
\end{array}\right]-\boldsymbol{\Pi}\left[\boldsymbol{\Psi}(\boldsymbol{\phi})\left[\begin{array}{c}
\operatorname{Re}\{\boldsymbol{\gamma}\} \\
\operatorname{Im}\{\boldsymbol{\gamma}\}
\end{array}\right]\right.} & \\
& \left.-\left[\begin{array}{c}
\operatorname{Re}\{\operatorname{vec}\{\hat{\mathbf{H}}\}\} \\
\operatorname{Im}\{\operatorname{vec}\{\hat{\mathbf{H}}\}\}
\end{array}\right]\right] .
\end{aligned}
$$

The matrix $\Pi$ is defined in (72) in Appendix A. Note that in (36), the term involving $\Pi$ serves as a correction term to the estimates for the unstructured model $\hat{\boldsymbol{\tau}}$ and $\hat{\boldsymbol{\nu}}$. Substituting (36) in (32) and minimizing with respect to $\left[\operatorname{Re}\{\boldsymbol{\gamma}\}^{\mathrm{T}} \operatorname{Im}\{\boldsymbol{\gamma}\}^{\mathrm{T}}\right]^{\mathrm{T}}$ yields

$$
\begin{array}{r}
{\left[\begin{array}{c}
\operatorname{Re}\{\hat{\hat{\gamma}}\} \\
\operatorname{Im}\{\hat{\hat{\gamma}}\}
\end{array}\right]=\left(\Psi^{\mathrm{T}}(\boldsymbol{\phi}) \boldsymbol{\Omega} \Psi(\boldsymbol{\phi})\right)^{-1}} \\
\cdot \boldsymbol{\Psi}^{\mathrm{T}}(\boldsymbol{\phi}) \boldsymbol{\Omega}\left[\begin{array}{c}
\operatorname{Re}\{\operatorname{vec}\{\hat{\mathbf{H}}\}\} \\
\operatorname{Im}\{\operatorname{vec}\{\hat{\mathbf{H}}\}\}
\end{array}\right] .
\end{array}
$$


The matrix $\boldsymbol{\Omega}$ is a Schur complement of the weighting matrix $\mathbf{W}$ and it is defined in (71) in Appendix A. Finally, introducing (36) and (37) to (32) we can derive the resulting problem

$$
\begin{aligned}
\hat{\hat{\boldsymbol{\phi}}}=\arg \min _{\boldsymbol{\phi}}\left\{\left[\begin{array}{l}
\operatorname{Re}\{\operatorname{vec}\{\hat{\mathbf{H}}\}\} \\
\operatorname{Im}\{\operatorname{vec}\{\hat{\mathbf{H}}\}\}
\end{array}\right]^{\mathrm{T}}(\boldsymbol{\Omega}-\boldsymbol{\Omega} \Psi(\boldsymbol{\phi})\right. \\
\left.\left.\left(\boldsymbol{\Psi}^{\mathrm{T}}(\boldsymbol{\phi}) \boldsymbol{\Omega} \boldsymbol{\Psi}(\boldsymbol{\phi})\right)^{-1} \boldsymbol{\Psi}^{\mathrm{T}}(\boldsymbol{\phi}) \boldsymbol{\Omega}\right)\left[\begin{array}{l}
\operatorname{Re}\{\operatorname{vec}\{\hat{\mathbf{H}}\}\} \\
\operatorname{Im}\{\operatorname{vec}\{\hat{\mathbf{H}}\}\}
\end{array}\right]\right\} .
\end{aligned}
$$

The problem as given in (38) provides estimates of the DOAs that are asymptotically equivalent to the ML estimates of the structured model. These estimates $\hat{\hat{\phi}}$ are introduced in (37) and (36) to improve the time-delay and Doppler frequency estimates $(\hat{\boldsymbol{\tau}}$ and $\hat{\boldsymbol{\nu}})$, which were derived in the first step using the spatially unstructured model. Thus, the refined estimates $\hat{\hat{\boldsymbol{\tau}}}$ and $\hat{\boldsymbol{\nu}}$ can be calculated. Applying the EXIP results in closed-form solutions for $\hat{\hat{\tau}}, \hat{\hat{\nu}}$ and $\hat{\hat{\gamma}}$ as given in (36) and (37). However, a search over the DOAs is required as given in (38). In Section IV-B we show that the problem in (38) is equivalent to a complex augmented SSF (cf. Theorem 2). In Section IV-C we prove that for a ULA the problem in (38) can be solved in closed-form (cf. Theorem 3). In Section IV-D it is shown that the WLS problem given in (32) provides a direct and natural way of taking array calibration errors into account.

\section{A. Complex Formulation of the DOA Estimation Problem}

The problem given in (38) can be transformed to a complex formulation based on $\mathbb{C}$-widely linear operations.

Theorem 2: The problem given in (38) is equivalent to a complex augmented SSF problem given as

$$
\begin{aligned}
\hat{\hat{\boldsymbol{\phi}}}=\arg \min _{\boldsymbol{\phi}}\left\{\operatorname { t r } \left(\mathbf{P}_{\tilde{\boldsymbol{\Omega}}^{\frac{1}{2}} \tilde{\mathbf{\Psi}}(\boldsymbol{\phi})}^{\perp} \tilde{\mathbf{\Omega}}^{\frac{\mathrm{H}}{2}}\left[\begin{array}{c}
\operatorname{vec}\{\hat{\mathbf{H}}\} \\
\operatorname{vec}\left\{\hat{\mathbf{H}}^{*}\right\}
\end{array}\right]\right.\right. & {\left.\left.\left[\begin{array}{c}
\operatorname{vec}\{\hat{\mathbf{H}}\} \\
\operatorname{vec}\left\{\hat{\mathbf{H}}^{*}\right\}
\end{array}\right]^{\mathrm{H}} \tilde{\mathbf{\Omega}}^{\frac{1}{2}}\right)\right\} }
\end{aligned}
$$

with

$$
\begin{array}{r}
\mathbf{P}_{\tilde{\boldsymbol{\Omega}}^{\frac{1}{2}} \tilde{\mathbf{\Psi}}(\boldsymbol{\phi})}^{\perp}=\mathbf{I}_{2 L M}-\tilde{\boldsymbol{\Omega}}^{\frac{1}{2}} \tilde{\mathbf{\Psi}}(\boldsymbol{\phi})\left(\tilde{\boldsymbol{\Psi}}^{\mathrm{H}}(\boldsymbol{\phi}) \tilde{\boldsymbol{\Omega}} \tilde{\mathbf{\Psi}}(\boldsymbol{\phi})\right)^{-1} \\
\tilde{\Psi}^{\mathrm{H}}(\boldsymbol{\phi}) \tilde{\boldsymbol{\Omega}}^{\mathrm{H}}
\end{array}
$$

where $\tilde{\Psi}(\boldsymbol{\phi}) \in \mathbb{C}^{2 L M \times 2 L}$ and $\tilde{\boldsymbol{\Omega}} \in \mathbb{C}^{2 L M \times 2 L M}$ are the complex augmented matrices of $\Psi(\phi)$ and $\Omega$, respectively.

Proof: The complex augmented SSF problem is derived in Appendix B.

The structure of (39) results from the fact that $\operatorname{vec}\{\hat{\mathbf{H}}\}$ is an improper complex random vector [37, p. 35]. Thus, (39) incorporates not only vec $\{\hat{\mathbf{H}}\}$ but also vec $\left\{\hat{\mathbf{H}}^{*}\right\}$ in the derived complex augmented notation based on $\mathbb{C}$-widely linear operations.

\section{B. Solution for Uniform Linear Arrays}

Here, we will derive an innovative reparametrization approach for a ULA based on IQML [25] that exploits the special Khatri-Rao structure of the complex augmented SSF problem (39) in order to achieve an efficient solution with low computational complexity. The problem (39) derived in Theorem
2 may be solved for arbitrary array geometries by adapting, for example, weighted subspace fitting (WSF) [38] or other SSF techniques such as [21], [22] to a complex augmented formulation in order to take into account the improper Gaussian problem at hand. In the case of a ULA, this adaptation to a complex augmented formulation could be performed based on the novel reparametrization approach presented in the theorem below.

Theorem 3: For a ULA the complex augmented SSF problem as given in Theorem 2 can be solved by iteratively evaluating a quadratic problem with linear constraints.

Proof: The proof follows two steps. First, we define a reparametrization of the problem given in (39). Second, we will show that the reparametrized problem can be solved by iteratively evaluating a quadratic problem with linear constraints. This second step is derived in Appendix C.

Consider a centrosymmetric ULA where the array elements are identical and equidistantly spaced by $\Delta$ along a line, with

$$
\mathbf{a}\left(\phi_{\ell}\right)=\mathrm{e}^{-\mathrm{j}\left(\frac{M-1}{2}\right) \mu_{\ell}}\left[1 \mathrm{e}^{\mathrm{j} \mu_{\ell}} \cdots \mathrm{e}^{\mathrm{j}(M-1) \mu_{\ell}}\right]^{\mathrm{T}} \in \mathbb{C}^{M \times 1}
$$

where $\mu_{\ell}=-\frac{2 \pi}{\lambda} \Delta \sin \left(\phi_{\ell}\right)$ and $\lambda$ denotes the wavelength. We define a Toeplitz matrix $\mathbf{B}_{\ell}$ with

$$
\mathbf{B}_{\ell}^{\mathrm{H}}=\left[\begin{array}{cccc}
b_{\ell, 1} & b_{\ell, 0} & & \mathbf{0} \\
& \ddots & \ddots & \\
\mathbf{0} & & b_{\ell, 1} & b_{\ell, 0}
\end{array}\right] \in \mathbb{C}^{(M-1) \times M}
$$

where $\mathbf{B}_{\ell}$ spans the nullspace of $\mathbf{a}\left(\phi_{\ell}\right)$ and we observe that

$$
\mathbf{B}_{\ell}^{\mathrm{H}} \mathbf{a}\left(\phi_{\ell}\right)=\mathbf{0}, \text { for } \ell=1, \ldots, L .
$$

The variables $b_{\ell, 0}$ and $b_{\ell, 1}$ denote the coefficients of a first-order polynomial $p_{\ell}(z)=z b_{\ell, 0}+b_{\ell, 1}$ with root $z=\mathrm{e}^{\mathrm{j} \mu_{\ell}}$. The coefficients of this first-order polynomial are placed in the vector

$$
\mathbf{b}_{\ell}=\left[b_{\ell, 0}, b_{\ell, 1}\right]^{\mathrm{T}} \in \mathbb{C}^{2 \times 1} .
$$

Now we can expand this reparametrization of $\mathbf{a}\left(\phi_{\ell}\right)$ to formulate a reparametrization of $\tilde{\Psi}(\phi)$ according to its Khatri-Rao structure. Hence, we define

$$
\begin{aligned}
\Xi^{\mathrm{H}}=\operatorname{block} \operatorname{diag}\left\{\mathbf{B}_{1}^{\mathrm{H}}, \ldots, \mathbf{B}_{L}^{\mathrm{H}}, \mathbf{B}_{1}^{\mathrm{T}}\right. & \left., \ldots, \mathbf{B}_{L}^{\mathrm{T}}\right\} \\
& \in \mathbb{C}^{2 L(M-1) \times 2 L M}
\end{aligned}
$$

such that

$$
\Xi^{\mathrm{H}} \tilde{\Psi}(\phi)=0
$$

where the operator block diag \{\} denotes a block diagonal matrix [36, p. 1360]. Since $\tilde{\boldsymbol{\Omega}}^{-\frac{1}{2}} \Xi \in \mathbb{C}^{2 L M \times 2 L(M-1)}$ has rank $2 L(M-1)$ and $\tilde{\boldsymbol{\Omega}}^{\frac{1}{2}} \tilde{\boldsymbol{\Psi}}(\boldsymbol{\phi}) \in \mathbb{C}^{2 L M \times 2 L}$ has rank $2 L$, it follows from (46) that $\tilde{\boldsymbol{\Omega}}^{-\frac{1}{2}} \boldsymbol{\Xi}$ spans the null space of $\tilde{\boldsymbol{\Omega}}^{\frac{1}{2}} \tilde{\boldsymbol{\Psi}}(\boldsymbol{\phi})$ and

$$
\begin{aligned}
\mathbf{P}_{\tilde{\boldsymbol{\Omega}}^{-\frac{1}{2}} \boldsymbol{\Xi}} & =\tilde{\boldsymbol{\Omega}}^{-\frac{1}{2}} \boldsymbol{\Xi}\left(\boldsymbol{\Xi}^{\mathrm{H}} \tilde{\boldsymbol{\Omega}}^{-\frac{\mathrm{H}}{2}} \tilde{\boldsymbol{\Omega}}^{-\frac{1}{2}} \boldsymbol{\Xi}\right)^{-1} \boldsymbol{\Xi}^{\mathrm{H}} \tilde{\boldsymbol{\Omega}}^{-\frac{\mathrm{H}}{2}} \\
& =\mathbf{P}_{\tilde{\boldsymbol{\Omega}}^{\frac{1}{2}} \tilde{\boldsymbol{\Psi}}(\boldsymbol{\phi})}^{\perp}
\end{aligned}
$$


Thus, we can reformulate the complex augmented SSF problem given in (39) replacing $\mathbf{P}_{\tilde{\mathbf{\Omega}}^{\frac{1}{2}} \tilde{\mathbf{\Psi}}(\boldsymbol{\phi})}^{\perp}$ by $\mathbf{P}_{\tilde{\boldsymbol{\Omega}}^{-\frac{1}{2}} \Xi}$ and we get

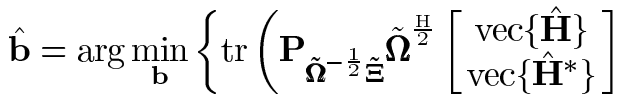

$$
\begin{aligned}
& \left.\left.\left[\begin{array}{c}
\operatorname{vec}\{\hat{\mathbf{H}}\} \\
\operatorname{vec}\left\{\hat{\mathbf{H}}^{*}\right\}
\end{array}\right]^{\mathrm{H}} \tilde{\mathbf{\Omega}}^{\frac{1}{2}}\right)\right\}
\end{aligned}
$$

where

$$
\mathbf{b}=\left[\mathbf{b}_{1}^{\mathrm{T}}, \ldots, \mathbf{b}_{L}^{\mathrm{T}}\right]^{\mathrm{T}} \in \mathbb{C}^{2 L \times 1} .
$$

In order to get a useful solution for $\mathbf{b}$ we must impose a constraint to avoid the trivial solution $\mathbf{b}=\mathbf{0}$. Without loss of generality we choose $\mathbf{b}_{\ell 0}=1 \forall_{\ell \in\{1, \ldots, L\}}$ for this non-triviality constraint. Thus, all the first-order polynomials $p_{\ell}(z)$ are to be monic [39] and $\mathbf{B}_{\ell}$ depends explicitly on a complex coefficient $b_{\ell, 1}$ and $\phi_{\ell}$ depends uniquely on $b_{\ell, 1}$, where $p_{\ell}(z)=z+b_{\ell, 1}$ and the root of this first-order polynomial is given by $z=-b_{\ell, 1}=$ $\mathrm{e}^{\mathrm{j} \mu_{\ell}}$. Finally, reformulating (48) and introducing the non-triviality constraint we get

$$
\begin{aligned}
& \hat{\mathbf{b}}=\arg \min _{\mathbf{b}}\left\{\operatorname { t r } \left(\left(\boldsymbol{\Xi}^{\mathrm{H}} \tilde{\boldsymbol{\Omega}}^{-1} \boldsymbol{\Xi}\right)^{-1} \boldsymbol{\Xi}^{\mathrm{H}}\left[\begin{array}{c}
\operatorname{vec}\{\hat{\mathbf{H}}\} \\
\operatorname{vec}\left\{\hat{\mathbf{H}}{ }^{*}\right\}
\end{array}\right]\right.\right. \\
& \left.\left.\left[\begin{array}{c}
\operatorname{vec}\{\hat{\mathbf{H}}\} \\
\operatorname{vec}\left\{\hat{\mathbf{H}}^{*}\right\}
\end{array}\right]^{\mathrm{H}} \boldsymbol{\Xi}\right)\right\} \\
& \text { s.t. } \mathbf{b}_{\ell 0}=1 \forall_{\ell \in\{1, \ldots, L\}} \text {. }
\end{aligned}
$$

Now, the problem as given in (50) can be solved by iteratively solving a quadratic problem with linear constraints. The proof is given in Appendix C. The DOAs can be directly determined as

$$
\hat{\hat{\phi}}_{\ell}=-\arcsin \left(\frac{\arg \left(-\hat{b}_{\ell 1}\right)}{\frac{2 \pi \Delta}{\lambda}}\right) .
$$

\section{Robustness to Calibration Errors}

The WLS problem given in (32) provides a direct and natural way of taking array calibration errors into account. This is accomplished by "regularization" of the WLS criterion, by introducing an appropriate modification of the weighting matrix [16]. Information on the array perturbations may be available from the manufacturer of the antenna array (e.g., given in terms of gain and phase tolerances) or from the results of several calibration measurements. The robustness of the EXIP WLS fit to array perturbations can be improved through the use of the following theorem.

Theorem 4: Suppose that for the mapping of the spatially unstructured onto the spatially structured model we have

$$
\left[\begin{array}{l}
\operatorname{Re}\{\operatorname{vec}\{\mathbf{H}\}\} \\
\operatorname{Im}\{\operatorname{vec}\{\mathbf{H}\}\}
\end{array}\right]=(\mathbf{\Psi}(\boldsymbol{\phi})+\overline{\mathbf{\Psi}})\left[\begin{array}{l}
\operatorname{Re}\{\boldsymbol{\gamma}\} \\
\operatorname{Im}\{\boldsymbol{\gamma}\}
\end{array}\right]
$$

where

$$
\overline{\mathbf{\Psi}}=\left[\begin{array}{cc}
\operatorname{Re}\left\{\mathbf{I}_{L} \square \overline{\mathbf{A}}\right\} & -\operatorname{Im}\left\{\mathbf{I}_{L} \square \overline{\mathbf{A}}\right\} \\
\operatorname{Im}\left\{\mathbf{I}_{L} \square \overline{\mathbf{A}}\right\} & \operatorname{Re}\left\{\mathbf{I}_{L} \square \overline{\mathbf{A}}\right\}
\end{array}\right]
$$

and $\overline{\mathbf{A}} \in \mathbb{C}^{M \times L}$ represents the matrix of array perturbations. Suppose that the columns of $\overline{\mathbf{A}}$, denoted as $\overline{\mathbf{a}}_{\ell}$, are modeled as zero-mean random vectors with known covariance. Then the "regularized" weighting matrix

$$
\mathbf{W}_{r}=\left[\begin{array}{cc}
\boldsymbol{\Omega}_{r}^{-1} & -\boldsymbol{\Omega}_{r}^{-1} \boldsymbol{\Pi}^{\mathrm{T}} \\
-\boldsymbol{\Pi} \boldsymbol{\Omega}_{r}^{-1} & \operatorname{Re}\left\{\mathbf{W}_{2}\right\}^{-1}+\boldsymbol{\Pi} \boldsymbol{\Omega}_{r}^{-1} \boldsymbol{\Pi}^{\mathrm{T}}
\end{array}\right]^{-1}
$$

with the "regularized" Schur complement $\boldsymbol{\Omega}_{r} \in \mathbb{C}^{2 L M \times 2 L M}$ will improve the robustness of the EXIP WLS fit.

Proof: The known covariance of $\operatorname{vec}\{\overline{\mathbf{A}}\}$ is

$$
\begin{array}{r}
\overline{\mathbf{R}}=\mathrm{E}\left[\left[\begin{array}{l}
\operatorname{Re}\{\operatorname{vec}\{\overline{\mathbf{A}}\}\} \\
\operatorname{Im}\{\operatorname{vec}\{\overline{\mathbf{A}}\}\}
\end{array}\right][\operatorname{Re}\{\operatorname{vec}\{\overline{\mathbf{A}}\}\} \operatorname{Im}\{\operatorname{vec}\{\overline{\mathbf{A}}\}\}]^{\mathrm{T}}\right] \\
\in \mathbb{R}^{2 L M \times 2 L M} .
\end{array}
$$

The difference between the nominal mapping and the mapping including array perturbations is the term $\bar{\Psi}\left[\operatorname{Re}\{\boldsymbol{\gamma}\}^{\mathrm{T}} \operatorname{Im}\{\boldsymbol{\gamma}\}^{\mathrm{T}}\right]^{\mathrm{T}}$ and its covariance can be written as

$$
\begin{aligned}
\overline{\boldsymbol{\Omega}}^{-1} & =\mathrm{E}\left[\overline{\boldsymbol{\Psi}}\left[\begin{array}{l}
\operatorname{Re}\{\boldsymbol{\gamma}\} \\
\operatorname{Im}\{\boldsymbol{\gamma}\}
\end{array}\right]\left[\operatorname{Re}\{\boldsymbol{\gamma}\}^{\mathrm{T}} \operatorname{Im}\{\boldsymbol{\gamma}\}^{\mathrm{T}}\right] \overline{\boldsymbol{\Psi}}^{\mathrm{T}}\right] \\
& =\overline{\boldsymbol{\Gamma}} \overline{\mathbf{R}} \overline{\boldsymbol{\Gamma}}^{\mathrm{T}}
\end{aligned}
$$

with

$$
\overline{\boldsymbol{\Gamma}}=\left[\begin{array}{cc}
\operatorname{Re}\{\boldsymbol{\Gamma}\} \otimes \mathbf{I}_{M} & -\operatorname{Im}\{\boldsymbol{\Gamma}\} \otimes \mathbf{I}_{M} \\
\operatorname{Im}\{\boldsymbol{\Gamma}\} \otimes \mathbf{I}_{M} & \operatorname{Re}\{\boldsymbol{\Gamma}\} \otimes \mathbf{I}_{M}
\end{array}\right] \in \mathbb{R}^{2 L M \times 2 L M} .
$$

Consequently, the "regularized" Schur complement is given by

$$
\boldsymbol{\Omega}_{r}=\left(\boldsymbol{\Omega}^{-1}+\overline{\mathbf{\Omega}}^{-1}\right)^{-1}
$$

which is introduced to $\mathbf{W}_{r}$.

In order to derive $\boldsymbol{\Omega}_{r}$ and $\overline{\boldsymbol{\Omega}}$ as defined in Theorem 4, an initial estimate of $\boldsymbol{\Gamma}$ and thus of $\boldsymbol{\gamma}$ is required. This initial estimate $\hat{\boldsymbol{\gamma}}_{0}$ can be obtained by an initial application of the EXIP algorithm without the regularized weighting [16]. Afterwards, one can set

$$
\boldsymbol{\Omega}_{r}\left(\hat{\boldsymbol{\gamma}}_{0}\right)=\left(\boldsymbol{\Omega}^{-1}+\overline{\boldsymbol{\Omega}}^{-1}\left(\hat{\boldsymbol{\gamma}}_{0}\right)\right)^{-1}
$$

and apply the EXIP with the regularized WLS approach.

\section{COMPutational Complexity}

The derived two-step approach provides a solution for the structured model $\boldsymbol{\theta}$ which involves reparameterizing the ML criterion in such a way that it admits a much simpler approximate initial solution using an unstructured model $\boldsymbol{\xi}$ in the first step which afterwards is refined by means of a WLS fit in the second step. We have shown that the DOA estimates and a refinement of the time-delay and Doppler frequency shift estimates in the second step can be derived jointly (for all $L$ ) based on a complex augmented SSF problem (cf. Theorem 2) or in case of a ULA even in closed-form (cf. Theorem 3).

The second step of our two-step approach does not involve any search since the estimates are obtained in closed-form. Thus, the overall computational complexity is only due to the first step. Essentially, this means that the proposed algorithm has a complexity similar to the one of an estimator of the unstructured model, which is much less than the complexity 
of a method that directly estimates the structured model. This complexity reduction is achieved regardless of the method of optimization (SAGE, Newton-type, etc.) considered to derive the solution. This is the basic idea of the application of the EXIP [16], [24].

For instance, if the algorithm proposed in Section III-B is used to estimate the parameters of the unstructured model $\boldsymbol{\xi}$, $2 L$ one-dimensional searches per iteration are needed, i.e., one search for the time-delay (26) and one for the Doppler frequency shift (27) of each wavefront. On the contrary, if a similar algorithm, also based on SAGE, were used to directly estimate the structured model $\boldsymbol{\theta}, 3 L$ one-dimensional searches per iteration would be needed as, besides the time-delays $\boldsymbol{\tau}$ and Doppler frequency shifts $\boldsymbol{\nu}$, the DOAs (azimuth angles $\phi$ ) also have to be estimated [13], [15].

Additionally, the proposed two-step approach provides a direct and natural way of taking array calibration errors into account (cf. Theorem 4), instead of applying autocalibration methods which would introduce even more complexity in the parameter estimation when directly solving for a structured model (cf. Section I).

\section{Simulation RESULTS}

In this section, the performance of the proposed two-step approach is assessed by computer simulations. For each parameter set, 2500 Monte Carlo runs are performed and we adopt the root mean square error (RMSE) and the Cramer-Rao lower bound (CRLB) for statistical analysis of the behavior of the proposed approach. The simulations consider the problem of time-delay and Doppler estimation of the LOSS of a GPS satellite in a multipath and RFI environment.

We assume a centrosymmetric ULA with $M=8$ isotropic sensor elements with $\Delta=\frac{\lambda}{2}$. We assume the antenna array response to be known and thus no "regularization" of the WLS weighting matrix is required as described in Section IV-C. The one-sided bandwidth of the signal is $B=1.023 \mathrm{MHz}$. For the known waveform $c(t)$ we consider a pseudo random (PR) sequence with Gold codes as used for the GPS C/A code with code period $T=1 \mathrm{~ms}, 1023$ chips per code period each with a time duration $T_{c}=977.52 \mathrm{~ns}$ [40]. The signal-to-noise ratio (SNR) denotes the LOSS-to-noise ratio and the effective SNR in $\mathrm{dB}$ can be obtained by [1]

$$
\mathrm{SNR}=\frac{\mathrm{C}}{\mathrm{N}_{0}}-10 \cdot \log _{10}(2 B)+10 \cdot \log _{10}\left(N_{c}\right)
$$

where $\frac{\mathrm{C}}{\mathrm{N}_{0}}$ in $\mathrm{dB}-\mathrm{Hz}$ denotes the carrier-to-noise density ratio and $N_{c} \in \mathbb{N}$ is the number of code periods within the observation period. The ratio $\frac{\mathrm{C}}{\mathrm{N}_{0}}$ is given with respect to the modulated L1 (Link 1) signal transmitted by a GPS satellite [40]. We assume $\frac{\mathrm{C}}{\mathrm{N}_{0}}=40.3 \mathrm{~dB}-\mathrm{Hz}[41]$ and $N_{c}=6$ which leads to $\mathrm{SNR}=-15.03 \mathrm{~dB}$.

In this analysis we consider a single reflective multipath as a function of its relative delay to the LOSS $(L=2)$. In the following, parameters with the subscript 1 refer to the LOSS and parameters with the subscript 2 refer to the reflection. The reflected multipath and the LOSS are considered to be in-phase, which means $\arg \left(\gamma_{1}\right)=\arg \left(\gamma_{2}\right)$ and the signal-to-multipath ratio (SMR) is $5 \mathrm{~dB}$. Further, we assume a temporally white
Gaussian RFI source with interference-to-signal ratio (ISR) of $40 \mathrm{~dB}$ which is responsible for the spatial covariance of the noise plus interference field. The RFI is broadband and uncorrelated with the signals and the noise. The DOAs for the LOSS and the multipath are $\phi_{1}=-30^{\circ}$ and $\phi_{2}=62^{\circ}$ and for the RFI signal is $\phi_{I}=10^{\circ}$, which is assumed to be a point source. Further, we define the relative time-delay between the LOSS and the multipath as $\Delta \tau=\frac{\left|\tau_{1}-\tau_{2}\right|}{T_{c}}$. We will consider two scenarios, denoted by scenario A and scenario B. In scenario A we assume that the relative Doppler shift between the LOSS and the multipath signal is $\Delta \nu=\left|\nu_{1}-\nu_{2}\right| T_{N_{c}}=0$, where $T_{N_{c}}=T N_{c}$ denotes the coherent observation time. In scenario $\mathrm{B}$ we analyze the case where $\Delta \nu=\left|\nu_{1}-\nu_{2}\right| T_{N_{c}}=0.5$.

For the SAGE algorithm, the convergence condition is considered satisfied if $\left|\hat{\tau}_{\ell}^{(k-1)}-\hat{\tau}_{\ell}^{(k)}\right|<0.1955 \mathrm{~ns} \forall_{\ell \in\{1, \ldots, L\}}$ and $\left|\hat{\nu}_{\ell}^{(k-1)}-\hat{\nu}_{\ell}^{(k)}\right|<0.01 \mathrm{~Hz} \forall_{\ell \in\{1, \ldots, L\}}$, or if $k>200$, where $k \in \mathbb{N}$ denotes the $k$ th iteration of the SAGE algorithm. In order to derive DOA estimates solving (38) we use the proposed reparametrization approach based on IQML. The convergence condition is satisfied either if $\left|\hat{\hat{\phi}}_{\ell}^{(q-1)}-\hat{\hat{\phi}}_{\ell}^{(q)}\right|<$ $0.01^{\circ} \forall_{\ell \in\{1, \ldots, L\}}$ or if $q>200$, where $q \in \mathbb{N}$ denotes the $q$-th iteration of the algorithm. All the convergence conditions above are selected to be at least smaller than 0.1 times the respective CRLB of the parameters for the given scenario. The maximum number of iterations is defined based on experience with simulations.

\section{A. Decision on the LOSS}

For the problem of time-delay and Doppler frequency estimation of the LOSS in GNSS applications, one has to decide which parameters refer to the LOSS after estimating $\boldsymbol{\xi}$ and $\boldsymbol{\theta}$. We propose to determine the parameter vector $\hat{\boldsymbol{\xi}}_{\ell}$ which refers to the LOSS by evaluating the decision criterion

$$
\max _{\ell=1, \ldots, L} \frac{\left\|\hat{\mathbf{Q}}^{-\frac{1}{2}} \hat{\mathbf{h}}_{\ell}\right\|_{2}^{2}}{M} \text {. }
$$

The term $\frac{\left\|\hat{\mathbf{Q}}^{-\frac{1}{2}} \hat{\mathbf{h}}_{\ell}\right\|_{2}^{2}}{M}$ is an estimate for $\left|\gamma_{\ell}\right|^{2}$. Based on the criterion given in (61) we automatically decide which $\hat{\boldsymbol{\theta}}_{\ell}$ refers to the LOSS, as the EXIP preserves the mapping between $\hat{\xi}_{\ell}$ and $\hat{\boldsymbol{\theta}}_{\ell}$. However, in case the two wavefronts are highly correlated for small $\Delta \tau, \Delta \nu=0$ and $\arg \left(\gamma_{1}\right)=\arg \left(\gamma_{2}\right)$, outliers in the decision on the LOSS can occur. These outliers are caused by non-convergence of the proposed reparametrization algorithm (when $q>200$ ) in case of model mismatch with respect to $L$. All these outliers are excluded from the error statistics which are presented in the following and the outlier percentage with respect to the total number of Monte Carlo runs is presented as a failure rate. Note that these failures only occur in scenario A with $\Delta \nu=0$ and not in scenario $\mathrm{B}$, where $\Delta \nu=0.5$. Scenario A can be considered a worst-case scenario with respect to correlation of the two wavefronts and also with respect to outliers in the LOSS decision.

In a practical implementation these failures can be detected for example by a consistency check over time of the DOA estimates of the LOSS and by tracking the LOSS based on its parameter estimates [35], [42], [43]. Such methods need to be 


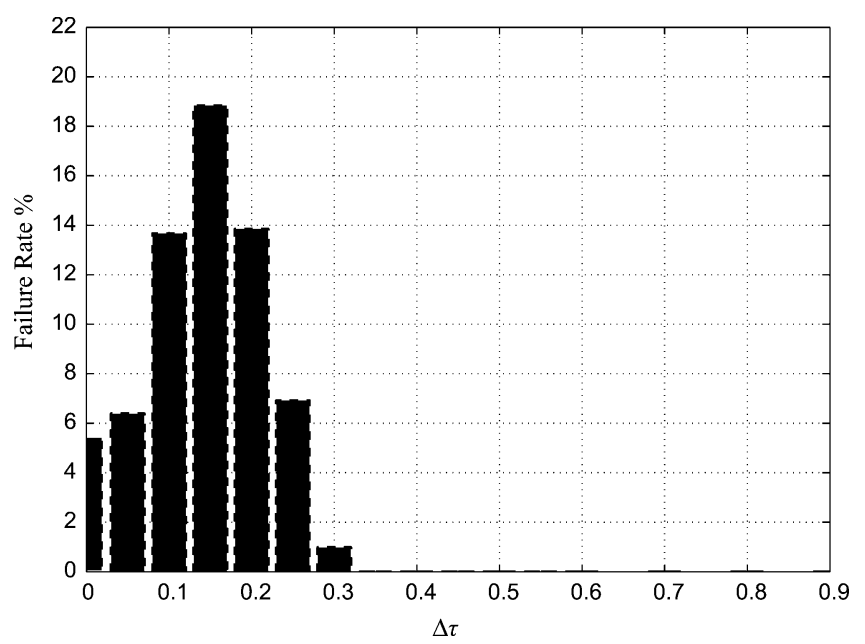

Fig. 1. Failure rate for the decision on the LOSS for scenario A.

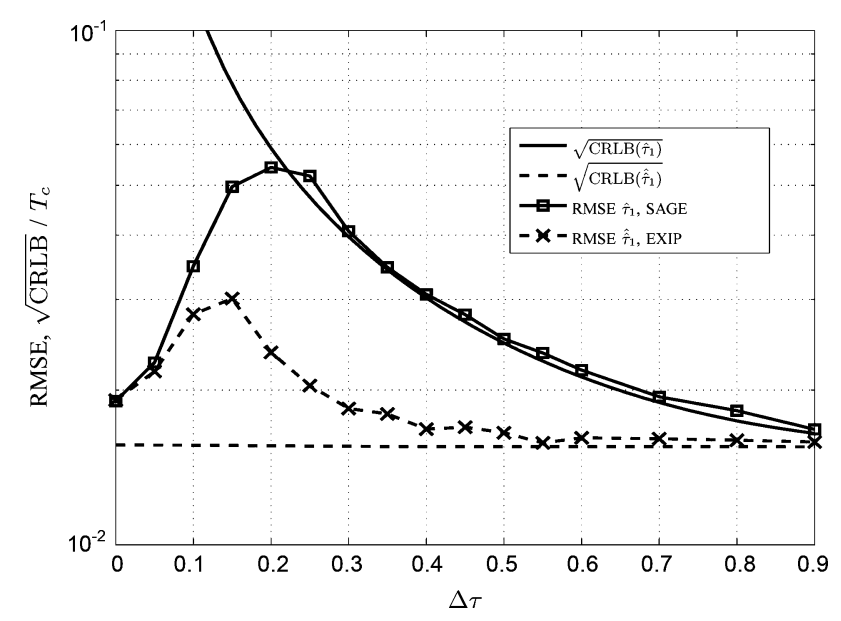

Fig. 2. RMSE of the time-delay of the LOSS for scenario A.

adopted for continuous LOSS tracking of spatial and synchronization parameters for GNSS applications [35].

\section{B. Scenario A}

For scenario A the relative Doppler shift is $\Delta \nu=0$. All other parameters are defined as outlined in the beginning of this section. In Fig. 1 the failure rate using the proposed reparametrization approach and the EXIP is shown for scenario A. In Fig. 2 to Fig. 7 the RMSE is depicted for all parameters of the unstructured and the structured model and for both the LOSS and the multipath signal with respect to the CRLB of the structured and unstructured models.

In Fig. 2 the SAGE algorithm for small $\Delta \tau$ becomes biased and the RMSE $\hat{\tau}_{1}$ is below $\sqrt{\operatorname{CRLB}\left(\hat{\tau}_{1}\right)}$, since the interference cancellation in the E-step (24) and (25) is not capable of separating the two signals if $\Delta \tau$ is very small [13]. In these cases the estimate of one hidden data space, $\hat{\mathbf{X}}_{1}$, is an estimate for a superposition of the two wavefronts and the other, $\hat{\mathbf{X}}_{2}$, includes only noise. This can be clearly seen in Figs. 5 and 6 where for small $\Delta \tau$ the RMSE $\hat{\tau}_{2}$ and RMSE $\hat{\nu}_{2}$ are increase drastically and are not close to $\sqrt{\operatorname{CRLB}\left(\hat{\tau}_{2}\right)}$ and $\sqrt{\operatorname{CRLB}\left(\hat{\nu}_{2}\right)}$. As the multipath signal for small $\Delta \tau$ can not be resolved by the SAGE algorithm

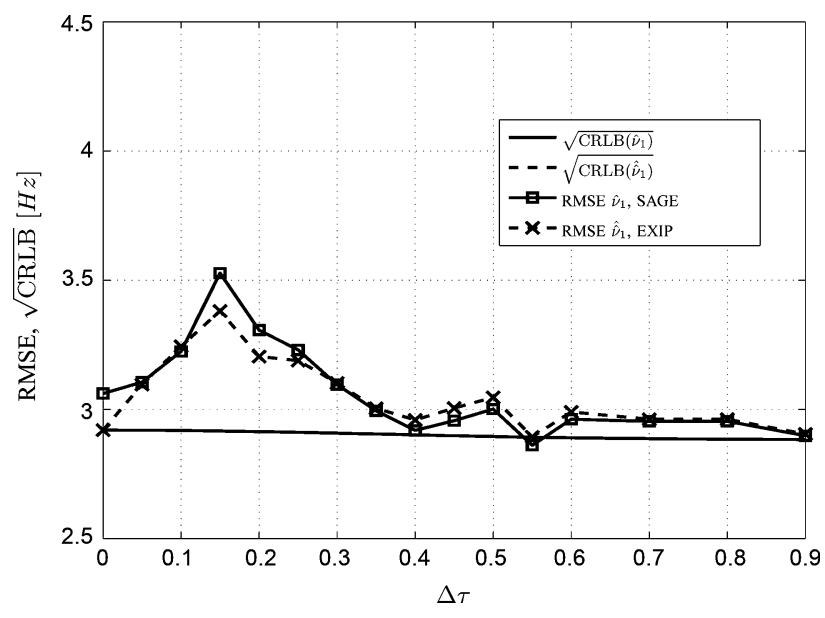

Fig. 3. RMSE of the Doppler frequency of the LOSS for scenario A.

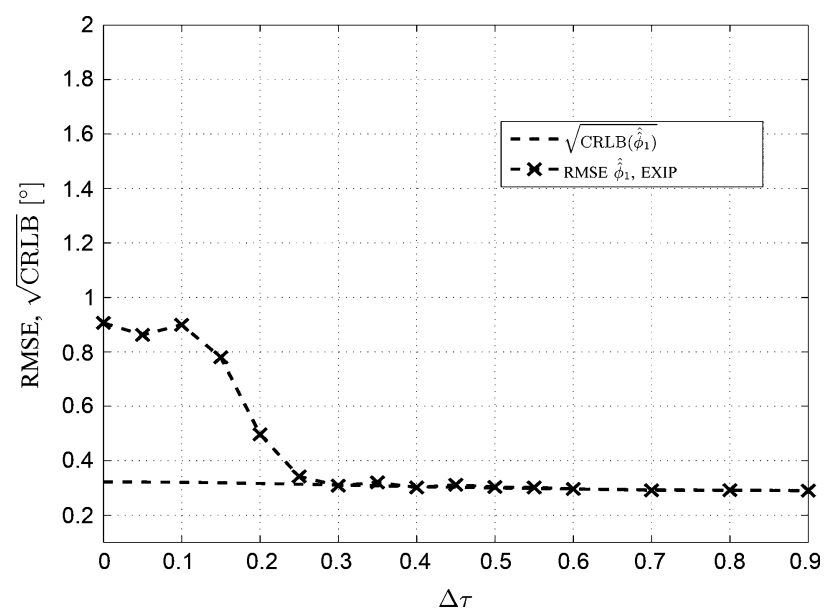

Fig. 4. RMSE of the azimuth angle of the LOSS for scenario A.

for the unstructured model, consequently the weighting matrix $\mathbf{W}$ which is calculated based on $\hat{\xi}$ and $\hat{\mathbf{Q}}$ does not provide the correct metric in order to achieve refinement of $\hat{\tau}_{2}$ and $\hat{\nu}_{2}$. This behavior can be inspected in Figs. 5 and 6 .

Even if the true DOAs of the $L$ wavefronts were available, the incorrect determination of $\mathbf{W}$ by $\hat{\xi}$ and $\hat{\mathbf{Q}}$ would inhibit refinement of $\hat{\tau}_{2}$ and $\hat{\nu}_{2}$ when the EXIP would be applied. However, considering the estimate of the time-delay $\hat{\tau}_{1}$ of the LOSS using the unstructured model, a significant improvement can be achieved by applying the EXIP while introducing the DOA estimates derived by the innovative IQML reparametrization. This can be seen in Fig. 2. The reason for this behavior is that the LOSS can be resolved by the SAGE algorithm for the unstructured model and thus $\mathbf{W}$ together with the DOA estimate $\hat{\hat{\phi}}_{1}$ achieve significant refinement of $\hat{\tau}_{1}$. For GNSS applications $\hat{\tau}_{1}$ is the most important parameter for positioning. Hence, the proposed two-step approach is very suitable for such applications.

In Fig. 3, the RMSE of $\hat{\nu}_{1}$ and $\hat{\nu}_{1}$ are shown. Notice that $\sqrt{\operatorname{CRLB}\left(\hat{\nu}_{1}\right)}$ and $\sqrt{\operatorname{CRLB}\left(\hat{\nu}_{1}\right)}$ are equivalent. Thus, no refinement in resolution of $\hat{\nu}_{1}$ can be expected by applying the EXIP. This is also given for $\hat{\nu}_{2}$ as $\sqrt{\operatorname{CRLB}\left(\hat{\nu}_{2}\right)}$ and $\sqrt{\operatorname{CRLB}\left(\hat{\hat{\nu}}_{2}\right)}$ are equivalent for scenario A as well, as shown in Fig. 6. 


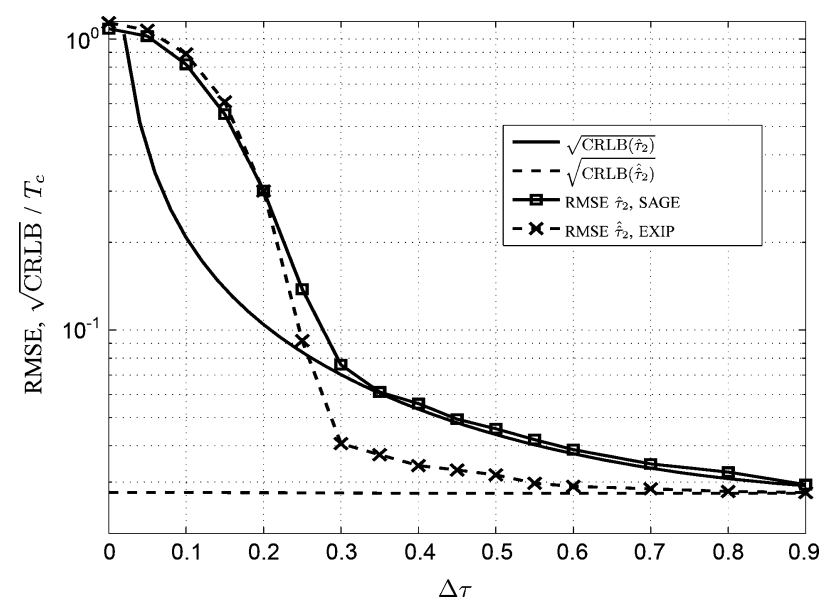

Fig. 5. RMSE of the time-delay of the multipath signal for scenario A.

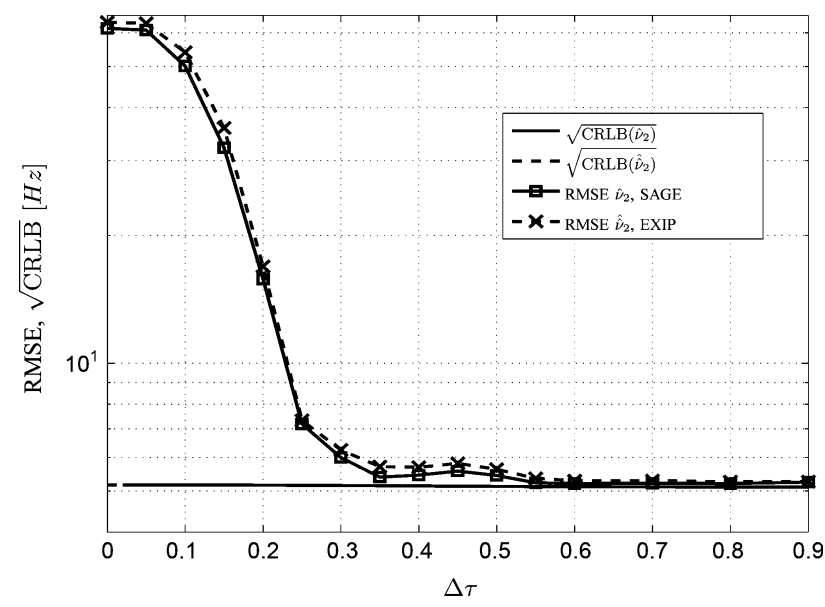

Fig. 6. RMSE of the Doppler frequency of the multipath signal for scenario A.

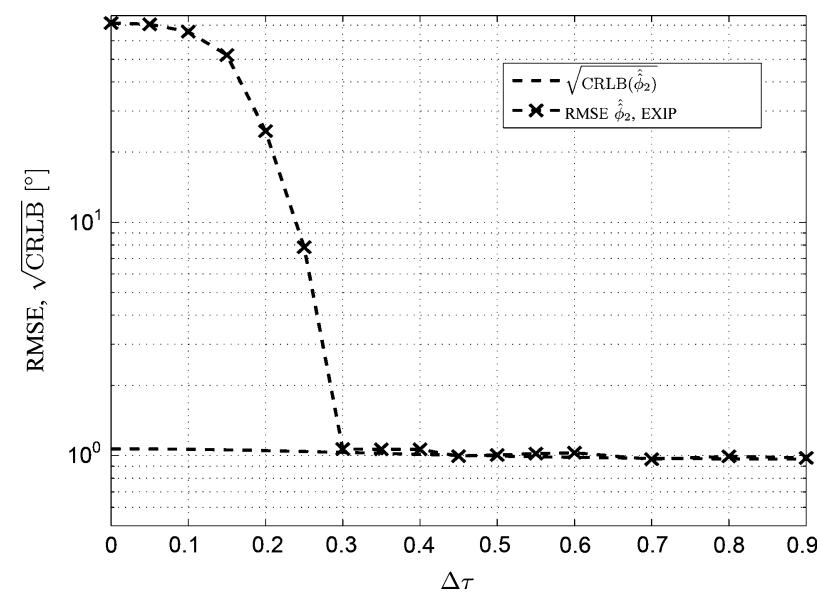

Fig. 7. RMSE of the azimuth angle of the multipath signal for scenario A.

\section{Scenario B}

In scenario B we choose the relative Doppler to be $\Delta \nu=0.5$. All other parameters are defined as outlined in the beginning of this section. In Fig. 8 to Fig. 10 the RMSE is depicted for parameters of the LOSS of unstructured and the structured model with respect to the CRLB of the structured and unstructured models.

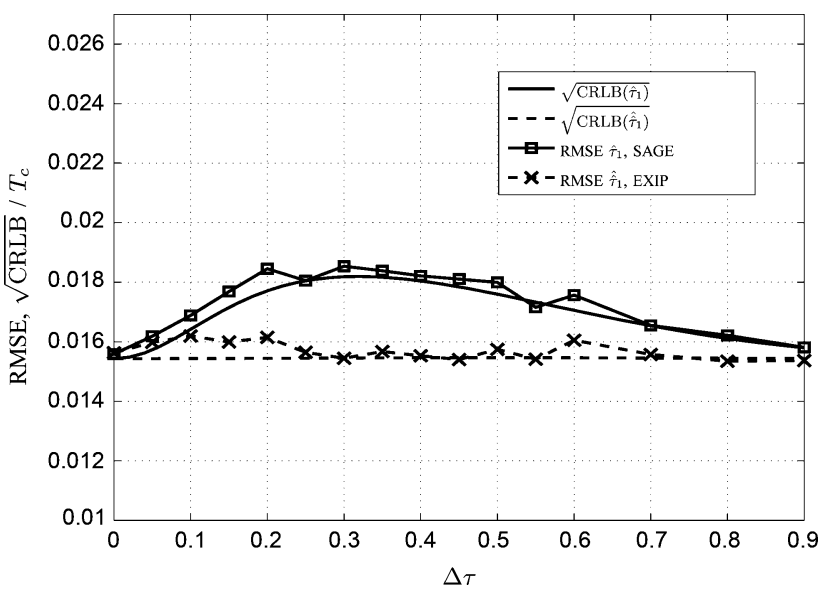

Fig. 8. RMSE of the time-delay of the LOSS for scenario B.

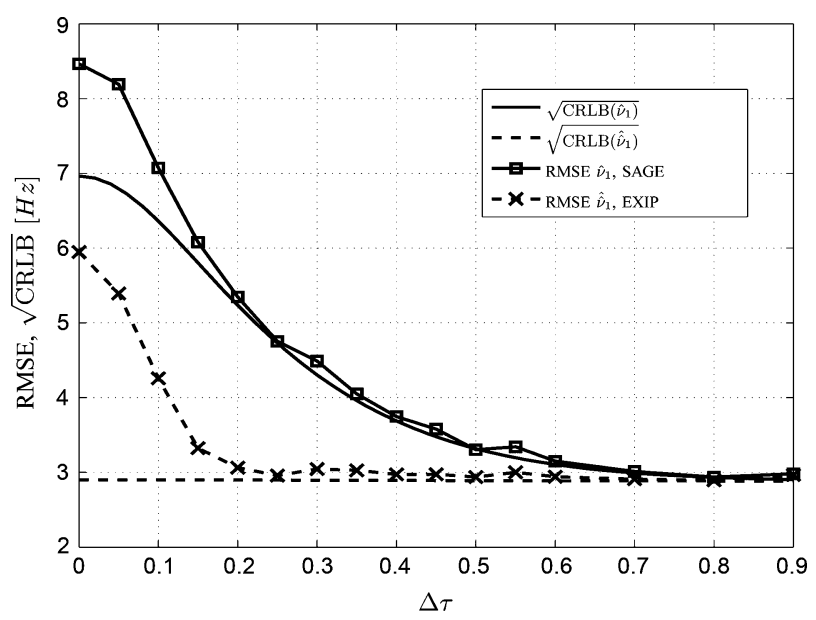

Fig. 9. RMSE of the Doppler frequency of the LOSS for scenario B.

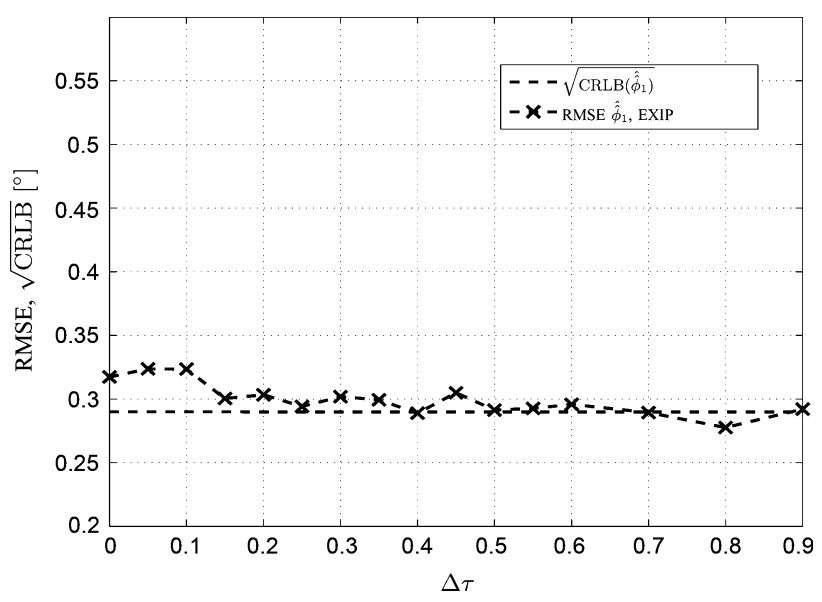

Fig. 10. RMSE of the azimuth angle of the LOSS for scenario B.

For this scenario no outliers in the decision of the LOSS occurred. The results with respect to the multipath signal are similar to the results presented for scenario A in Section VI-B.

In Figs. 8 and 9 it can be observed that the estimator for the parameters of the unstructured model at best should be an efficient ML estimator in order to achieve near optimum refinement 
of the estimates in the second step. Thus, an asymptotically efficient high-resolution method to solve the MLE of the first step, such as the SAGE algorithm, provides a reasonable tradeoff between estimation performance and computational complexity for the application at hand [13], [15].

As shown in Fig. 8, the RMSE of $\hat{\tau}_{1}$ and $\hat{\tau}_{1}$ approach $\sqrt{\operatorname{CRLB}\left(\hat{\tau}_{1}\right)}$ and $\sqrt{\operatorname{CRLB}\left(\hat{\hat{\tau}}_{1}\right)}$, respectively. The LOSS is resolved by the SAGE algorithm and hence an improvement in resolution of $\hat{\tau}_{1}$ is achieved by applying the EXIP with the DOA estimate of the LOSS. Also for the Doppler estimate $\hat{\nu}_{1}$ a refinement can be achieved which can be seen in Fig. 9.

In Fig. 10 the RMSE of $\hat{\hat{\phi}}_{1}$ for the proposed reparametrization approach is given. We can see that $\hat{\phi}_{1}$ can be derived with high accuracy and thus a refinement of $\hat{\tau}_{1}$ and $\hat{\nu}_{1}$ can be accomplished by applying the EXIP, as shown in Figs. 8 and 9.

\section{CONCLUSION}

In this paper, a two-step approach based on the EXIP is proposed in order to achieve estimates of a spatially structured model in an unknown spatially colored field. This two-step approach involves significantly less complexity than directly solving for the parameters of the spatially structured model and yet its performance approaches the structured model CRLB. Furthermore, it provides a direct and natural way of taking into account array antenna calibration errors without a significant increase in computational complexity.

The WLS fit in the second step involves a search only for the DOAs of the impinging wavefronts. We have proven that the resulting DOA estimation problem is equivalent to a complex augmented SSF problem and we have derived an efficient low-complexity solution for a ULA based on an innovative reparametrization approach that exploits the special structure of this complex augmented SSF problem. In order to achieve robustness of the EXIP to errors in the array response model, a novel "regularization" of the weighting matrix of the WLS was defined based on a "regularized" Schur complement.

Simulation results for time-delay and Doppler frequency estimation in a GPS application were presented for a two-path case and confirm that the proposed two-step approach attains the CRLB of the spatially structured model and thus is asymptotically efficient.

\section{APPENDIX A}

\section{DERIVATION OF THE WeIGHTING MATRIX W}

The weighting matrix $\mathbf{W}$ can be calculated following the derivation of the FIM for the spatially unstructured model as given in [44, p. 70 et seq.] and extending it to handle the Doppler frequencies of the wavefronts:

$$
\mathbf{W}=\left[\begin{array}{ccc}
\operatorname{Re}\left\{\mathbf{W}_{1}\right\} & -\operatorname{Im}\left\{\mathbf{W}_{1}\right\} & \operatorname{Re}\left\{\mathbf{W}_{3}\right\} \\
\operatorname{Im}\left\{\mathbf{W}_{1}\right\} & \operatorname{Re}\left\{\mathbf{W}_{1}\right\} & \operatorname{Im}\left\{\mathbf{W}_{3}\right\} \\
\operatorname{Re}\left\{\mathbf{W}_{3}\right\}^{\mathrm{T}} & \operatorname{Im}\left\{\mathbf{W}_{3}\right\}^{\mathrm{T}} & \operatorname{Re}\left\{\mathbf{W}_{2}\right\}
\end{array}\right]
$$

with

$$
\mathbf{W}_{1}=2\left((\mathbf{C} \odot \mathbf{D})(\mathbf{C} \odot \mathbf{D})^{\mathrm{H}}\right) \otimes \hat{\mathbf{Q}}^{-1}
$$

$$
\begin{aligned}
\mathbf{W}_{2}= & 2\left(\boldsymbol{\Upsilon}^{\mathrm{H}} \boldsymbol{\Upsilon}\right) \odot\left(\left[\mathbf{e}_{2} \mathbf{e}_{2}\right] \otimes\left(\hat{\mathbf{H}}^{\mathrm{H}} \hat{\mathbf{Q}}^{-1} \hat{\mathbf{H}}\right)\right) \\
\mathbf{W}_{3}= & {\left[\begin{array}{c}
2\left(\mathbf{e}_{2}^{\mathrm{T}} \otimes\left(\hat{\mathbf{Q}}^{-1} \hat{\mathbf{H}}\right)\right) \operatorname{diag}\left\{\left(\mathbf{c}\left(\hat{\tau}_{1}\right) \odot \mathbf{d}\left(\hat{\nu}_{1}\right)\right)^{\mathrm{H}} \boldsymbol{\Upsilon}\right\} \\
\vdots \\
2\left(\mathbf{e}_{2}^{\mathrm{T}} \otimes\left(\hat{\mathbf{Q}}^{-1} \hat{\mathbf{H}}\right)\right) \operatorname{diag}\left\{\left(\mathbf{c}\left(\hat{\tau}_{\ell}\right) \odot \mathbf{d}\left(\hat{\nu}_{\ell}\right)\right)^{\mathrm{H}} \boldsymbol{\Upsilon}\right\} \\
\vdots \\
2\left(\mathbf{e}_{2}^{\mathrm{T}} \otimes\left(\hat{\mathbf{Q}}^{-1} \hat{\mathbf{H}}\right)\right) \operatorname{diag}\left\{\left(\mathbf{c}\left(\hat{\tau}_{L}\right) \odot \mathbf{d}\left(\hat{\nu}_{L}\right)\right)^{\mathrm{H}} \boldsymbol{\Upsilon}\right\}
\end{array}\right] }
\end{aligned}
$$

where $\otimes$ denotes the Kronecker operator and

$$
\begin{aligned}
\mathbf{e}_{2}= & {\left[\begin{array}{ll}
1 & 1
\end{array}\right]^{\mathrm{T}} } \\
\mathbf{\Upsilon}= & {\left[\frac{\partial \mathbf{c}\left(\hat{\imath}_{1}\right)}{\partial \hat{\tau}_{1}} \odot \mathbf{d}\left(\hat{\nu}_{1}\right) \cdots \frac{\partial \mathbf{c}\left(\hat{\tau}_{\ell}\right)}{\partial \hat{\tau}_{\ell}} \odot \mathbf{d}\left(\hat{\nu}_{\ell}\right) \cdots \frac{\partial \mathbf{c}\left(\hat{\tau}_{L}\right)}{\partial \hat{\tau}_{L}} \odot \mathbf{d}\left(\hat{\nu}_{L}\right)\right.} \\
& \left.\mathbf{c}\left(\hat{\tau}_{1}\right) \odot \frac{\partial \mathbf{d}\left(\hat{\nu}_{1}\right)}{\partial \hat{\nu}_{1}} \cdots \mathbf{c}\left(\hat{\tau}_{\ell}\right) \odot \frac{\partial \mathbf{d}\left(\hat{\nu}_{\ell}\right)}{\partial \hat{\nu}_{\ell}} \cdots \mathbf{c}\left(\hat{\tau}_{L}\right) \odot \frac{\partial \mathbf{d}\left(\hat{\nu}_{L}\right)}{\partial \hat{\nu}_{L}}\right] \\
\frac{\partial \mathbf{c}\left(\hat{\tau}_{\ell}\right)}{\partial \hat{\tau}_{\ell}}= & {\left[-\left.\frac{d c(t)}{d t}\right|_{\left(T_{s}-\hat{\tau}_{\ell}\right)}, \ldots,-\left.\frac{d c(t)}{d t}\right|_{\left(n T_{s}-\hat{\tau}_{\ell}\right)}, \ldots,-\left.\frac{d c(t)}{\mathrm{d} t}\right|_{\left(N T_{S}-\hat{\tau}_{\ell}\right)}\right]^{\mathrm{T}} } \\
\frac{\partial \mathbf{d}\left(\hat{\nu}_{\ell}\right)}{\partial \hat{\nu}_{\ell}}= & {\left[\mathrm{j} 2 \pi T_{s} \mathrm{e}^{\mathrm{j} 2 \pi \hat{\nu}_{\ell} T_{s}}, \ldots, \mathbf{j} 2 \pi n T_{s} \mathrm{e}^{\mathrm{j} 2 \pi \hat{\nu}_{\ell} n T_{s}}, \ldots, \mathrm{j} 2 \pi N T_{s} \mathrm{e}^{\mathrm{j} 2 \pi \hat{\nu}_{\ell} N T_{S}}\right]^{\mathrm{T}} . }
\end{aligned}
$$

Note that $\mathbf{W}$ is nonsingular and positive semidefinite, so $\mathbf{W}=$ $\mathbf{W}^{\mathrm{T}}$ and $-\operatorname{Im}\left\{\mathbf{W}_{1}\right\}^{\mathrm{T}}=\operatorname{Im}\left\{\mathbf{W}_{1}\right\}$. The inverse of $\mathbf{W}(\mathrm{Ba}-$ nachiewicz inversion formula [45, p. 165]) can be calculated using

$$
\mathbf{W}^{-1}=\left[\begin{array}{cc}
\boldsymbol{\Omega}^{-1} & -\boldsymbol{\Omega}^{-1} \boldsymbol{\Pi}^{\mathrm{T}} \\
-\boldsymbol{\Pi} \boldsymbol{\Omega}^{-1} & \operatorname{Re}\left\{\mathbf{W}_{2}\right\}^{-1}+\boldsymbol{\Pi} \boldsymbol{\Omega}^{-1} \Pi^{\mathrm{T}}
\end{array}\right]
$$

where $\boldsymbol{\Omega}$ denotes the Schur complement of block $\operatorname{Re}\left\{\mathbf{W}_{2}\right\}$ in W

$$
\begin{aligned}
\boldsymbol{\Omega}= & {\left[\begin{array}{ll}
\operatorname{Re}\left\{\mathbf{W}_{1}\right\} & -\operatorname{Im}\left\{\mathbf{W}_{1}\right\} \\
\operatorname{Im}\left\{\mathbf{W}_{1}\right\} & \operatorname{Re}\left\{\mathbf{W}_{1}\right\}
\end{array}\right] } \\
& -\left[\begin{array}{l}
\operatorname{Re}\left\{\mathbf{W}_{3}\right\} \\
\operatorname{Im}\left\{\mathbf{W}_{3}\right\}
\end{array}\right] \operatorname{Re}\left\{\mathbf{W}_{2}\right\}\left[\operatorname{Re}\left\{\mathbf{W}_{3}\right\}^{\mathrm{T}} \operatorname{Im}\left\{\mathbf{W}_{3}\right\}^{\mathrm{T}}\right]
\end{aligned}
$$

and

$$
\boldsymbol{\Pi}=\operatorname{Re}\left\{\mathbf{W}_{2}\right\}^{-1}\left[\operatorname{Re}\left\{\mathbf{W}_{3}\right\}^{\mathrm{T}} \operatorname{Im}\left\{\mathbf{W}_{3}\right\}^{\mathrm{T}}\right] .
$$

As $\mathbf{W}$ and $\operatorname{Re}\left\{\mathbf{W}_{2}\right\}$ are nonsingular and symmetric, it follows that the Schur complement $\boldsymbol{\Omega}$ is nonsingular and symmetric as well $[45$, p. 165$]$. The matrix $\boldsymbol{\Omega}^{-1}$ can be interpreted as an estimate of the covariance of $\left[\operatorname{Re}\{\operatorname{vec}\{\hat{\mathbf{H}}\}\}^{\mathrm{T}}, \operatorname{Im}\{\operatorname{vec}\{\hat{\mathbf{H}}\}\}^{\mathrm{T}}\right]^{\mathrm{T}}$ for the case where $\tau$ and $\nu$ are unknown.

\section{APPENDIX B}

PROOF OF THEOREM 2

We define two unitary real-to-complex transformation matrices $\mathbf{T}_{1}$ and $\mathbf{T}_{2}$ following [37, p. 31 et seq.]:

$$
\begin{aligned}
& \mathbf{T}_{1}=\frac{1}{\sqrt{2}}\left[\begin{array}{cc}
\mathbf{I}_{L M} & \mathrm{j} \mathbf{I}_{L M} \\
\mathbf{I}_{L M} & -\mathrm{j} \mathbf{I}_{L M}
\end{array}\right] \in \mathbb{C}^{2 L M \times 2 L M} \\
& \mathbf{T}_{2}=\frac{1}{\sqrt{2}}\left[\begin{array}{cc}
\mathbf{I}_{L} & \mathrm{j} \mathbf{I}_{L} \\
\mathbf{I}_{L} & -\mathrm{j} \mathbf{I}_{L}
\end{array}\right] \in \mathbb{C}^{2 L \times 2 L} .
\end{aligned}
$$

We introduce the terms $\mathbf{T}_{1} \mathbf{T}_{1}^{\mathrm{H}}$ and $\mathbf{T}_{2} \mathbf{T}_{2}^{\mathrm{H}}$ to (38) according to the dimensions of $\Psi(\phi), \quad \Omega$ and $\left[\operatorname{Re}\{\operatorname{vec}\{\mathbf{H}\}\}^{\mathrm{T}}, \operatorname{Im}\{\operatorname{vec}\{\mathbf{H}\}\}^{\mathrm{T}}\right]^{\mathrm{T}} \quad$ without altering the original problem. Then we can restructure the resulting 
problem by introducing the complex augmented matrices $\mathbf{T}_{1} \boldsymbol{\Omega} \mathbf{T}_{1}^{\mathrm{H}}=\mathbf{T}_{1} \boldsymbol{\Omega}^{\mathrm{T}} \mathbf{T}_{1}^{\mathrm{H}}=\frac{1}{2} \boldsymbol{\mathbf { \Omega }} \in \mathbb{C}^{2 L M \times 2 L M}$ and $\mathbf{T}_{1} \boldsymbol{\Psi}(\boldsymbol{\phi}) \mathbf{T}_{2}^{\mathrm{H}}=\tilde{\boldsymbol{\Psi}}(\boldsymbol{\phi}) \in \mathbb{C}^{2 L M \times 2 L}$. Thus

$$
\begin{aligned}
\tilde{\boldsymbol{\Omega}} & =\left[\begin{array}{ll}
\tilde{\boldsymbol{\Omega}}_{11} & \tilde{\boldsymbol{\Omega}}_{12} \\
\tilde{\boldsymbol{\Omega}}_{21} & \tilde{\boldsymbol{\Omega}}_{22}
\end{array}\right] \\
& =\left[\begin{array}{cc}
2 \mathbf{W}_{1}-\mathbf{W}_{3} \operatorname{Re}\left\{\mathbf{W}_{2}\right\}^{-1} \mathbf{W}_{3}^{\mathrm{H}} & -\mathbf{W}_{3} \operatorname{Re}\left\{\mathbf{W}_{2}\right\}^{-1} \mathbf{W}_{3}^{\mathrm{T}} \\
-\mathbf{W}_{3}^{*} \operatorname{Re}\left\{\mathbf{W}_{2}\right\}^{-1} \mathbf{W}_{3}^{\mathrm{H}} & 2 \mathbf{W}_{1}^{*}-\mathbf{W}_{3}^{*} \operatorname{Re}\left\{\mathbf{W}_{2}\right\}^{-1} \mathbf{W}_{3}^{\mathrm{T}}
\end{array}\right]
\end{aligned}
$$

$$
\tilde{\mathbf{\Psi}}(\boldsymbol{\phi})=\left[\begin{array}{cc}
\mathbf{I}_{L} \square \mathbf{A} & \mathbf{0} \\
\mathbf{0} & \mathbf{I}_{L} \square \mathbf{A}^{*}
\end{array}\right]
$$

and the complex augmented vector of the real composed vector $\left[\operatorname{Re}\{\operatorname{vec}\{\hat{\mathbf{H}}\}\}^{\mathrm{T}} \operatorname{Im}\{\operatorname{vec}\{\hat{\mathbf{H}}\}\}^{\mathrm{T}}\right]^{\mathrm{T}}$ is

$$
\frac{1}{\sqrt{2}}\left[\begin{array}{c}
\operatorname{vec}\{\hat{\mathbf{H}}\} \\
\operatorname{vec}\left\{\hat{\mathbf{H}}^{*}\right\}
\end{array}\right]=\mathbf{T}_{1}\left[\begin{array}{l}
\operatorname{Re}\{\operatorname{vec}\{\hat{\mathbf{H}}\}\} \\
\operatorname{Im}\{\operatorname{vec}\{\hat{\mathbf{H}}\}\}
\end{array}\right] \in \mathbb{C}^{1 \times 2 L M} .
$$

Now we can write (38) using (75)-(77) in a complex augmented formulation

$$
\begin{aligned}
\hat{\hat{\boldsymbol{\phi}}}=\arg \min _{\boldsymbol{\phi}}\left\{\frac { 1 } { 4 } [ \begin{array} { c } 
{ \operatorname { v e c } \{ \hat { \mathbf { H } } \} } \\
{ \operatorname { v e c } \{ \hat { \mathbf { H } } ^ { * } \} }
\end{array} ] ^ { \mathrm { H } } \left(\tilde{\boldsymbol{\Omega}}-\tilde{\boldsymbol{\Omega}}^{\mathrm{H}} \tilde{\mathbf{\Psi}}(\boldsymbol{\phi})\right.\right. \\
\left.\left.\left(\tilde{\Psi}^{\mathrm{H}}(\boldsymbol{\phi}) \tilde{\boldsymbol{\Omega}} \tilde{\Psi}(\boldsymbol{\phi})\right)^{-1} \tilde{\boldsymbol{\Psi}}^{\mathrm{H}}(\boldsymbol{\phi}) \tilde{\boldsymbol{\Omega}}\right)\left[\begin{array}{c}
\operatorname{vec}\{\hat{\mathbf{H}}\} \\
\operatorname{vec}\left\{\hat{\mathbf{H}}^{*}\right\}
\end{array}\right]\right\} .
\end{aligned}
$$

Based on (78) the complex augmented SSF problem can be derived.

\section{APPENDIX C}

PART OF PROOF OF THEOREM 3

We define

$$
\Xi^{\mathrm{H}}\left[\begin{array}{c}
\operatorname{vec}\{\hat{\mathbf{H}}\} \\
\operatorname{vec}\left\{\hat{\mathbf{H}}^{*}\right\}
\end{array}\right]=\boldsymbol{\Phi}\left[\begin{array}{c}
\mathbf{b} \\
\mathbf{b}^{*}
\end{array}\right]=\boldsymbol{\Phi} \boldsymbol{\beta}
$$

with

$$
\begin{aligned}
\mathbf{\Phi}= & \text { block diag }\left\{\left[\mathbf{J}_{1} \hat{\mathbf{h}}_{\mathbf{1}} \mathbf{J}_{2} \hat{\mathbf{h}}_{1}\right], \ldots,\left[\mathbf{J}_{1} \hat{\mathbf{h}}_{L} \mathbf{J}_{2} \hat{\mathbf{h}}_{L}\right],\left[\mathbf{J}_{1} \hat{\mathbf{h}}_{1} \mathbf{J}_{2} \hat{\mathbf{h}}_{1}\right]^{*}, \ldots,\right. \\
& {\left.\left[\mathbf{J}_{\mathbf{1}} \hat{\mathbf{h}}_{L} \mathbf{J}_{2} \hat{\mathbf{h}}_{L}\right]^{*}\right\} \in \mathbb{C}^{2 L(M-1) \times 4 L} } \\
\mathbf{J}_{1}= & {\left[\mathbf{0} \mathbf{I}_{M-1}\right] \in \mathbb{R}^{(M-1) \times M} } \\
\mathbf{J}_{2}= & {\left[\mathbf{I}_{M-1} \mathbf{0}\right] \in \mathbb{R}^{(M-1) \times M} . }
\end{aligned}
$$

Now, we can reformulate (50) and we obtain

$$
\begin{gathered}
\min _{\mathbf{b}} \boldsymbol{\beta}^{\mathrm{H}}(\mathbf{b}) \boldsymbol{\Phi}^{\mathrm{H}}\left(\boldsymbol{\Xi}^{\mathrm{H}}(\mathbf{b}) \tilde{\boldsymbol{\Omega}}^{-1} \boldsymbol{\Xi}(\mathbf{b})\right)^{-1} \boldsymbol{\Phi} \boldsymbol{\beta}(\mathbf{b}), \\
\text { s.t. } \quad \mathbf{G}^{\mathrm{H}} \boldsymbol{\beta}(\mathbf{b})=\mathbf{e}_{2 L}
\end{gathered}
$$

with

$$
\begin{aligned}
\mathbf{G} & =\mathbf{I}_{2 L} \otimes\left[\begin{array}{l}
1 \\
0
\end{array}\right] \in \mathbb{R}^{4 L \times 2 L} \\
\mathbf{e}_{2 L} & =[1 \ldots 1]^{\mathrm{T}} \in \mathbb{R}^{2 L \times 1} .
\end{aligned}
$$

In order to solve (83) we establish the following IQML approach based on [25] and [39]. The term $\boldsymbol{\Theta}\left(\mathbf{b}_{(q-1)}\right)=$ $\left(\boldsymbol{\Xi}^{\mathrm{H}}\left(\mathbf{b}_{(q-1)}\right) \tilde{\boldsymbol{\Omega}}^{-1} \boldsymbol{\Xi}\left(\mathbf{b}_{(q-1)}\right)\right)^{-1}$ is fixed based on the estimates of $\mathbf{b}$ of the previous iteration step denoted by $q-1$. Then we solve the quadratic problem given in (83) in order to obtain a new estimate for $\mathbf{b}$. Thus, for each iteration step we have to solve

$$
\begin{gathered}
\min _{\mathbf{b}_{(q)}} \boldsymbol{\beta}^{\mathrm{H}}\left(\mathbf{b}_{(q)}\right) \boldsymbol{\Phi}^{\mathrm{H}} \boldsymbol{\Theta}\left(\mathbf{b}_{(q-1)}\right) \boldsymbol{\Phi} \boldsymbol{\beta}\left(\mathbf{b}_{(q)}\right), \\
\text { s.t. } \quad \mathbf{G}^{\mathrm{H}} \boldsymbol{\beta}\left(\mathbf{b}_{(q)}\right)=\mathbf{e}_{2 L} .
\end{gathered}
$$

The problem given in (86) is a quadratic problem with linear constraints and thus admits the following closed-form solution:

$$
\begin{aligned}
\boldsymbol{\beta}\left(\mathbf{b}_{(q)}\right)= & \left(\boldsymbol{\Phi}^{\mathrm{H}} \boldsymbol{\Theta}\left(\mathbf{b}_{(q-1)}\right) \boldsymbol{\Phi}\right)^{-1} \\
& \mathbf{G}\left(\mathbf{G}^{\mathrm{H}}\left(\boldsymbol{\Phi}^{\mathrm{H}} \boldsymbol{\Theta}\left(\mathbf{b}_{(q-1)}\right) \boldsymbol{\Phi}\right)^{-1} \mathbf{G}\right)^{-1} \mathbf{e}_{2 L} .
\end{aligned}
$$

The final problem (87) is evaluated iteratively until convergence with respect to a convergence criterion is achieved. Initialization can performed with e.g., $\mathbf{b}_{(0)}=[1,0,1,0, \ldots, 1,0]^{\mathrm{T}}$.

\section{REFERENCES}

[1] M. S. Braasch and A. J. van Dierendonck, "GPS receiver architecture and measurements," Proc. IEEE, vol. 87, no. 1, Jan. 1999.

[2] R. D. J. van Nee, "Spread-spectrum code and carrier synchronization errors caused by multipath and interference," IEEE Trans. Aerosp. Electron. Syst., vol. 29, no. 4, Oct. 1993.

[3] Global Positioning System: Theory and Applications, B. W. Parkinson and J. J. Spilker, Eds. Washington, DC: Progress in Astronautics and Aeronautics, 1996, vol. 1.

[4] R. D. J. van Nee and P. C. Fenton, "The multipath estimating delay lock loop approaching theoretical accuracy limits," in Proc. IEEE Position Location and Navigat. Symp., Las Vegas, NV, Apr. 1994.

[5] J. Soubielle, I. Fijalkow, P. Duvaut, and A. Bibaut, "GPS positioning in a multipath environment," IEEE Trans. Signal Process., vol. 50, no. 1, Jan. 2002.

[6] P. Closas, C. Fernandes-Prades, and J. A. Fernandes-Rubio, "A particle filtering tracking algorithm for GNSS synchronization using laplacets method," in Proc. 33rd IEEE Int. Conf. Acoust., Speech Signal Process. (ICASSP), Las Vegas, NV, Apr. 2008.

[7] B. Krach, M. Lentmaier, and P. Robertson, "Joint Baysian positioning and multipath mitigation in GNSS," in Proc. 33rd IEEE Int. Conf. Acoust., Speech Signal Process. (ICASSP), Las Vegas, NV, Apr. 2008.

[8] G. Seco-Granados, J. A. Fernández-Rubio, and C. Fernández-Prades, "ML estimator and hybrid beamformer for multipath and interference mitigation in GNSS receivers," IEEE Trans. Signal Process., vol. 53, no. 3, Mar. 2005

[9] J. Selva-Vera, "An efficient Newton-type method for the computation of ML estimators in a uniform linear array," IEEE Trans. Signal Process., vol. 53, no. 6, Jun. 2005.

[10] M. G. Amin, L. Zhao, and A. R. Lindsey, "Subspace array processing for the suppression of FM jamming in GPS receivers," IEEE Trans. Aerosp. Electron. Syst., vol. 40, no. 1, Jan. 2004.

[11] A. L. Swindlehurst, "Time delay and spatial signature estimation using known asynchronous signals," IEEE Trans. Signal Process., vol. 46, no. 2, Feb. 1998.

[12] G. Seco, A. L. Swindlehurst, and D. Astely, "Exploiting antenna arrays for synchronization," in Signal Processing Advances in Wireless and Mobile Communications, Volume 2: Trends in Single- and Multi-User Systems, G. B. Giannakis, P. Stoica, Y. Hua, and L. Tong, Eds. Englewood Cliffs, NJ: Prentice-Hall, 2000, pp. 403-430.

[13] B. H. Fleury, M. Tschudin, R. Heddergott, D. Dahlhaus, and K. I. Pedersen, "Channel parameter estimation in mobile radio environments using the SAGE algorithm," IEEE J. Sel. Areas Commun., vol. 17, no. 3, Mar. 1999

[14] M. Tschudin, C. Brunner, T. Krupjuhn, M. Haardt, and J. A. Nossek, "Comparison between unitary ESPRIT and SAGE for 3-D channel sounding," in Proc. IEEE Veh. Technol. Conf. (VTC), 1999.

[15] F. Antreich, J. A. Nossek, and W. Utschick, "Maximum likelihood delay estimation in a navigation receiver for aeronautical applications," Aerosp. Sci. Technol., vol. 12, no. 3, Apr. 2008, doi: 10.1016/j.ast. 2007.06.005.

[16] A. L. Swindlehurst and P. Stoica, "Maximum likelihood methods in radar array signal processing," Proc. IEEE, vol. 86, no. 2, Feb. 1998.

[17] A. L. Swindlehurst and T. Kailath, "A performance analysis of subspace-based methods in presence of model errors: Part I-the music algorithm," IEEE Trans. Signal Process., vol. 41, no. 9, Sep. 1993.

[18] A. L. Swindlehurst and T. Kailath, "A performance analysis of subspace-based methods in presence of model errors: Part II-multidimensional algorithms," IEEE Trans. Signal Process., vol. 41, no. 9, Sep. 1993. 
[19] M. Viberg and A. L. Swindlehurst, "Analysis of the combined effects of finite samples and model errors on array processing performance," IEEE Trans. Signal Process., vol. 42, no. 12, Dec. 1994.

[20] K. Pensel, H. Aroudaki, and J. A. Nossek, "Calibration of smart antennas in a GSM network," in Proc. Signal Process. Adv. Wireless Commun. (SPAWC), Annapolis, MD, USA, May 1999.

[21] M. Viberg and A. L. Swindlehurst, "A baysian approach to auto-calibration for parametric array sigal processing," IEEE Trans. Signal Process., vol. 42, no. 12, Dec. 1994.

[22] M. Jansson, A. L. Swindlehurst, and B. Ottersten, "Weighted subspace fitting for general array error models," IEEE Trans. Signal Process., vol. 46, no. 9, Sep. 1998.

[23] M. T. Ivrlac and J. A. Nossek, "Toward a circuit theory of communication," IEEE Trans. Circuits Syst., vol. 57, no. 7, 2010.

[24] P. Stoica and T. Söderström, "On reparametrization of loss functions used in estimation and the invariance principle," Signal Process., vol. 17, pp. 383-387, Aug. 1989

[25] P. Stoica and K. Sharman, "Maximum likelihood methods for direction-of-Arrival estimation," IEEE Trans. Acoust., Speech Signal Process., vol. 38, no. 7, Jul. 1990.

[26] A. Richter, "On the estimation of radio channel parameters: Models and algorithms (RIMAX)," Ph.D., TU-Ilmenau, Ilmenau, Germany, 2005.

[27] J. A. Fessler and A. O. Hero, "Space-alternating generalized expectation-maximization algorithm," IEEE Trans. Signal Process., vol. 42, no. 10, Oct. 1994

[28] F. A. Dietrich, A Tutorial on Channel Estimation with SAGE 2006, Tech. Rep. TUM-LNS-TR-06-03.

[29] H. Akaike, "A new look at the statistical model identification," IEEE Trans. Autom. Control, vol. 19, no. 6, 1974.

[30] J. Rissanen, "Modeling by shortest data description," Automatica, vol. $14,1978$.

[31] M. Wax and T. Kailath, "Detection of signals by information theoretic criteria," IEEE Trans. Acoust., Speech, Signal Process., vol. 33, no. 2, 1985.

[32] B. Ottesten, M. Viberg, P. Stoica, and A. Nehorai, "Exact and large sample maximum likelihood techniques for parameter estimation and detection in array processing," in Radar Array Processing, S. Haykin, J. Litva, and T. J. Shepherd, Eds. New York: Springer-Verlag, 1993, pp. 99-151.

[33] P. Stoica and M. Cedervall, "Detection tests for array processing in unknown correlated noise fields," IEEE Trans. Signal Process., vol. 45, no. 9, 1997.

[34] Q. Wu and K. M. Wong, "Array signal number detection for coherent and uncoherent signals in unknown noise environment," in Proc. IEEE Int. Conf. Acoust., Speech Signal Process. (ICASSP), Adelaide, Australia, Apr. 1994

[35] W. Wang, T. Jost, and A. Dammann, "Estimation and modelling of NLOS time-variant multipath for localization channel model in mobile radios," in Proc. IEEE Global Commun. Conf. (GLOBECOM), Miami, FL, Dec. 2010.

[36] H. L. V. Trees, Optimum Array Processing. Detection, Estimation and Modulation Theory, Part IV. New York: Wiley Interscience, 2002.

[37] P. J. Schreier and L. L. Scharf, Statistical Signal Processing of Complex-Valued Data, The Theory of Improper and Noncircular Signals. Cambridge: Cambridge Univ. Press, 2010.

[38] M. Viberg and B. Ottersten, "Sensor array processing based on subspace fitting," IEEE Trans. Signal Process., vol. 39, no. 5, May 1991.

[39] Y. Bresler and A. Macovski, "Exact maximum likelihood parameter estimation of superimposed exponential signals in noise," IEEE Trans. Acoust., Speech Signal Process. , vol. 34, no. 5, Oct. 1986.

[40] Navstar GPS Space Segment/Navigation User Interfaces, Navstar GPS Joint Program Office (SMC/GP) 2006.

[41] Minimum Operational Performance Standards (MOPS) for Global Positioning (GPS)/Wide Area Augmentation System (WAAS) Airborne Equipment, RTCA 2001.

[42] C. K. Sword, M. Simaan, and E. W. Kamen, "Multiple target angle tracking using sensor array outputs," IEEE Trans. Aerosp. Electron. Syst., vol. 26, no. 2, 1990.

[43] Y. Bar-Shalom, X. R. Li, and T. Kirubarajan, Estimation with Applications to Tracking and Navigation: Theory Algorithms and Software. New York: Wiley, 2001.
[44] G. Seco-Granados, "Antenna Arrays for Multipath and Interference Mitigation in GNSS Receivers," Ph.D., Dep. Signal Signal Theory and Commun., Univ. Politècnica de Catalunya, , Spain, 2000.

[45] , F. Zhang, Ed., The Schur Complement and its Applications. New York: Springer Sci. Business+Media Inc., 2005.

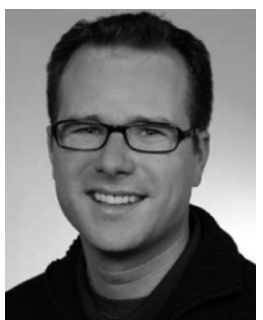

Felix Antreich (M'06) received the diploma in electrical engineering from the Munich University of Technology (TUM), Munich, Germany, in 2003.

$\mathrm{He}$ is currently pursuing the Ph.D. degree in electrical engineering at the TUM. Since July 2003, he has been an Associate Researcher with the Department of Navigation, Institute of Communications and Navigation of the German Aerospace Center (DLR), Wessling-Oberpfaffenhofen. His research interests include sensor array signal processing for global navigation satellite systems (GNSS) and wireless communications, estimation theory and signal design for synchronization, and GNSS.

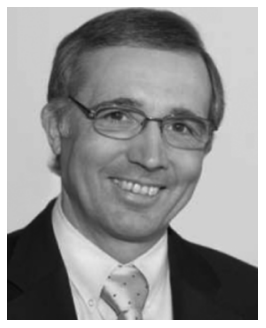

Josef A. Nossek (S'72-M'74-SM'81-F'93) received the Dipl.-Ing. and the Dr. techn. degrees in electrical engineering from the University of Technology in Vienna, Austria, in 1974 and 1980, respectively.

In 1974, he joined Siemens AG, Munich, Germany, as a Member of Technical staff, in 1978 he became Supervisor, and since 1980, he was Head of the Department. In 1987 he was promoted to Head of all radio systems design. Since 1989, he has been Full Professor for circuit theory and signal processing at the Munich University of Technology (TUM), Munich, Germany, where he teaches undergraduate and graduate courses on circuit and systems theory and signal processing and leads research on signal processing algorithms for communications.

Dr. Nossek was President Elect, President, and Past President of the IEEE Circuits and Systems Society in 2001, 2002, and 2003, respectively. He was Vice President of Verband der Elektrotechnik, Elektronik und Informationstechnik e.V. (VDE) in 2005 and 2006, President of VDE 2007 and 2008, and is again Vice President of VDE in 2009 and 2010. His awards include the ITG Best Paper Award 1988, the Mannesmann Mobilfunk (now Vodafone) Innovations award 1998, the Award for Excellence in Teaching from the Bavarian Ministry for Science, Research and Art in 1998, and the Golden Jubilee Medal of the IEEE Circuits and Systems Society for Outstanding Contributions to the Society in 1999. In 2008, he received the Education Award of the IEEE Circuits and Systems Society and the Order of Merit from the Federal Republic of Germany. Since 2009, he has been a Member of the National Academy of Engineering Sciences of Germany (acatech)

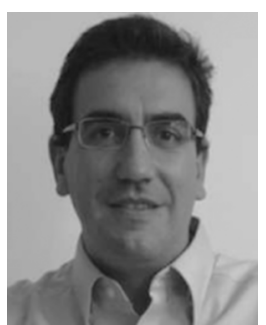

Gonzalo Seco-Granados (S'97-M'02-SM'08) received the Ph.D. degree in telecommunication engineering from the Universitat Politècnica de Catalunya (UPC), Barcelona, Spain, in 2000, and the M.B.A. degree from IESE-University of Navarra, Barcelona, Spain, in 2002.

During 2002-2005, he was Member of the Technical staff with the RF Payload Division, European Space Research and Technology Center (ESTEC), European Space Agency, Noordwijk, The Netherlands, where he was involved in the Galileo project. He led the activities concerning navigation receivers and indoor positioning for GPS and Galileo. Since 2006, he has been an Associate Professor with the Department of Telecommunications and Systems Engineering, Universitat Autònoma de Barcelona, Barcelona. He has been principal investigator of more than 10 national and international research projects. His research interests include signal processing for wireless communications and navigation, array signal processing, location-based communications, synchronization, estimation theory, and resource allocation.

Dr. Seco-Granados received two Best Ph.D. degree Thesis awards from the UPC and the Spanish Association of Telecommunication Engineers, as 
well as the Best Presentation Award at the ION-GPS'2003 conference. He was Co-Guest Editor for a Special Issue of the IEEE Signal Processing MAGAZINE and is an Associate Editor of the Hindawi International Journal of Navigation and Observation. Since March 2007, he has been coordinator of the Telecommunications Engineering degree and, since March 2008, has been the Director of the Chair of Technology and Knowledge Transfer UAB Research Park-Santander.

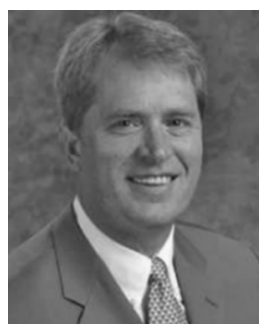

A. Lee Swindlehurst (S'83-M'84-SM'99-F'04) received the B.S. (summa cum laude) and M.S. degrees in electrical engineering from Brigham Young University, Provo, UT, in 1985 and 1986, respectively, and the Ph.D. degree in electrical engineering from Stanford University, Stanford, CA, in 1991 .

From 1986 to 1990, he was with ESL, Inc., Sunnyvale, $\mathrm{CA}$, where he was involved in the design of algorithms and architectures for several radar and sonar signal processing systems. He was on the faculty of the Department of Electrical and Computer Engineering, Brigham Young University, from 1990 to 2007, where he was a Full Professor and served as Department Chair from 2003 to 2006. During 1996-1997, he held a joint appointment as a Visiting Scholar at both Uppsala University, Uppsala, Sweden, and at the Royal Institute of Technology, Stockholm, Sweden. From 2006 to 2007, he was on leave working as Vice President of Research for ArrayComm LLC, San Jose, CA. He is currently a Professor of Electrical Engineering and Computer Science, University of California at Irvine. His research interests include sensor array signal processing for radar and wireless communications, detection and estimation theory, and system identification. He has more than 190 publications in these areas.

Dr. Swindlehurst is a past Secretary of the IEEE Signal Processing Society, past Editor-in-Chief of the IEEE JOURNAL OF SELECTED TOPICS IN SIGNAL PROCESSING and past member of the Editorial Boards for the EURASIP Journal on Wireless Communications and Networking, IEEE SignAL PROCESSING Magazine, and the IEEE Transactions on Signal Processing. He is a recipient of several paper awards: the 2000 IEEE W. R. G. Baker Prize Paper Award, the 2006 and 2010 IEEE Signal Processing Society's Best Paper Award, the 2006 IEEE Communications Society Stephen O. Rice Prize in the Field of Communication Theory. He is coauthor of a paper that received the IEEE Signal Processing Society Young Author Best Paper Award in 2001. 